\title{
ON THE ESTABLISHMENT OF THE THERMODYNAMIC SCALE OF TEMPERATURE BY MEANS OF THE CONSTANT-PRESSURE THERMOMETER.
}

\author{
By Edgar Buckingham.
}

\section{CONTENTS.}

Page.

I. Introduction $\ldots \ldots \ldots \ldots \ldots \ldots \ldots \ldots \ldots \ldots \ldots \ldots \ldots \ldots \ldots \ldots \ldots \ldots$

2. Theory of the porous-plug experiment................... 243

3. Theory of the constant-volume thermometer................ 247

4. Theory of the constant-pressure thermometer................. 249

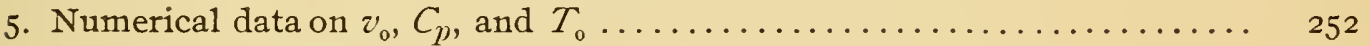

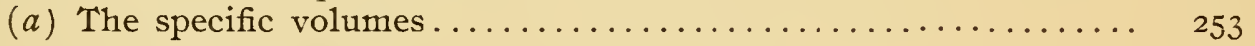

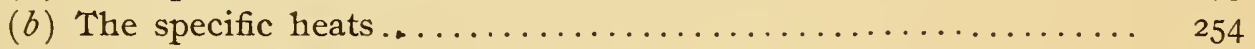

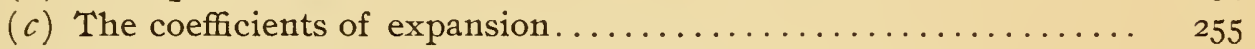

6. Experimental data on the Joule-Thomson effect................ 256

7. Application of the law of corresponding states ................ 258

8. Remarks on the equation $\mu=f(\tau) \ldots \ldots \ldots \ldots \ldots \ldots \ldots \ldots \ldots \ldots \ldots$

9. Integration of the equations of the constant-pressure thermometer . . . . . 266

Io. Values found for the thermodynamic temperature of the ice point ...... 269

II. Corrections of the constant-pressure hydrogen and nitrogen thermometers between the ice and steam points................. 274

12. Computation of the corrections for temperatures outside the fundamental

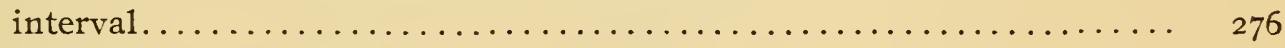

I3. Constant-pressure corrections for hydrogen and nitrogen outside the fundamental interval ........ .................. 279

I4. The boiling point of sulphur $\ldots \ldots \ldots \ldots \ldots \ldots \ldots \ldots \ldots \ldots \ldots \ldots \ldots \ldots \ldots \ldots$

I5. The relation of the constant-volume and constant-pressure scales...... 282

I6. Assumptions regarding the variation of $K$ with temperature......... 284

I7. Corrections of the constant-volume nitrogen thermometer......... 287

I8. Corrections of the constant-volume hydrogen thermometer.......... 289

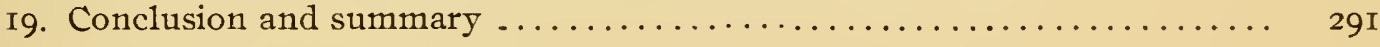

\section{INTRODUCTION.}

Two principal methods of gas thermometry are in use. In the constant-volume thermometer a mass of gas is kept at constant volume and its pressure observed at the melting point of ice, at the 
condensing point of steam $^{1}$, and at the temperature to be determined. If these three pressures be denoted by $p_{0}, p_{100}$, and $p$, the centigrade temperature according to the scale of this thermometer is by definition

$$
t_{v}=\mathrm{IOO} \frac{p-p_{0}}{p_{100}-p_{0}}
$$

The numerical value thus assigned to a given temperature depends slightly on the initial pressure and is somewhat different for different gases. There are thus various "constant-volume scales," all agreeing at $\mathrm{O}^{\circ}$ and $100^{\circ} \mathrm{C}$ but differing at other points, so that the value assigned to any given fixed temperature other than the two standard temperatures is slightly different on different scales.

In the constant-pressure thermometer a mass of gas is kept at constant pressure, and its volume observed at the two standard temperatures and at the temperature to which a numerical value is to be assigned. If these volumes be $v_{0}, v_{100}$, and $v$, the centigrade temperature according to the scale of this thermometer is by definition

$$
t_{p}=\mathrm{IOO} \frac{v-v_{0}}{v_{100}-v_{0}}
$$

The resulting value depends somewhat on the magnitude of the constant pressure and on the nature of the gas used, so that there are various "constant-pressure scales."

If the thermometric gases employed be always far from condensation so that they follow Boyle's law approximately, all these scales are nearly identical; the numerical value assigned to any given temperature is nearly the same, no matter which scale is used. The adoption of any particular scale is arbitrary and, except in work of the highest precision, one is as good as another.

In exact thermometry it is customary to refer all temperatures to. the international "normal scale" of the constant-volume hydrogen thermometer in which $p_{0}=$ the pressure of I meter of mercury. This thermometer can be used down to very low temperatures, though for practical reasons it is convenient to modify the scale by using a larger initial pressure $p_{0}$. Helium also is used for very low temperatures and its scale is nearly identical with the hydrogen scale.

\footnotetext{
'In what follows it is always to be understood that in making these observations the ice and the steam are at standard atmospheric pressure.
} 
At moderately high temperatures the materials which have been used for thermometer bulbs appear to become permeable to or to react with hydrogen, so that exact work with the hydrogen thermometer becomes impossible at temperatures above $300^{\circ} \mathrm{C}$. Helium exhibits some of the same phenomena as hydrogen. For higher temperatures nitrogen is found satisfactory. Air has also been used, and for medium temperature ranges is entirely satisfactory. These four gases are the only ones that have been in common use.

The constant-pressure method has fallen somewhat into disrepute and has not been used much in recent years. It has, however, certain practical advantages at high temperatures where there is danger of softening the bulb, since it obviates the necessity of applying a variable compensating pressure to the outside of the bulb, and it has recently ${ }^{2}$ been recommended for high temperatures by Barus.

There are thus in practical use a number of different gas-thermometer scales using different gases, different initial pressures, and two different principles. In precision measurements, the differences between these scales become very sensible, and it is important that we should know the relations subsisting among them so as to be able to reduce measurements made in terms of any one to some standard scale.

The normal constant-volume hydrogen scale, as maintained at the International Bureau of Weights and Measures, is the standard usually adopted, and temperatures are stated in terms of that scale; but, as has been said, the hydrogen thermometer can not be used at even moderately high temperatures, so that direct comparison is possible only for what must now be regarded as a very limited range. Nitrogen, on the other hand, though suitable for work at high temperatures, will not do for very low temperatures, because its critical temperature is too high. There thus appears to be no gas known which is satisfactory throughout the entire range of temperatures accessible to gas thermometry. Instead, therefore, of attempting to refer all measurements to a single gas scale (a process which it seems must inevitably involve a considerable extrapolation at one end or the other), it is preferable to refer all measurements to some more general scaleone which is valid for all temperatures.

${ }^{2}$ Rapports Congr. Int. de Physique, Paris, I9oo; 1, p. I48. 
By means of the two laws of thermodynamics any desired number of such scales might be defined, but there is one particular one, Lord Kelvin's thermodynamic scale, which has a practical advantage over all others in that it may be made to agree with all the gas scales in use as closely as they agree with one another, so that for most purposes its adoption does not complicate matters by adding a new scale to the number of those already in use. Lord Kelvin's scale may be defined as follows: Let an ideal reversible thermal engine work between two reservoirs of heat. Let the quantities of heat taken in or given out by the engine in the parts of its cycle when it is in contact with the two reservoirs be $Q_{1}$ and $Q_{2}$. Then the ratio $\theta_{1} \theta_{2}$ of the temperatures of the two reservoirs is by definition the same as the ratio of $Q_{1}$ to $Q_{2}$, or

$$
\frac{\theta_{1}}{\theta_{2}}=\frac{Q_{1}}{Q_{2}}
$$

This equation defines only the ratio of two temperatures and not their numerical values. If the further condition be imposed that the temperatures of the melting point of ice and the condensing point of steain shall differ by $100^{\circ}$, the scale is completely determined. By Carnot's theorem, the ratio $Q_{1} / Q_{2}$ depends only on the temperatures and not on the particular nature of the heat engine; hence the thermometric scale thus defined is independent of the properties of any particular substance. When the scale of temperature is defined in this way, the temperature of the melting point of ice, or shortly "the ice point," is approximately $273^{\circ}$, and therefore that of the "steam point" is $373^{\circ}$. If we denote by $\theta_{0}$ the exact value of the thermodynamic termperature of the ice point, we may define the "centigrade thermodynamic temperature" $t_{\theta}$ of any other point, by the equation

$$
t_{\theta}=\theta-\theta_{0}
$$

This amounts to saying that the centigrade thermodynamic temperature is the number of thermodynamic centigrade degrees from the ice point to the given temperature to be 1 11mbered. It is this centigrade thermodynamic scale which is nearly identical with the centigrade scales of the varions gas thermometers. 
The coefficient of pressure, which is always nearly $\frac{I}{273}$, is defined by the equation

$$
\beta=\frac{p_{100}-p_{0}}{\text { IOO } p_{0}}
$$

If we let $\frac{\mathrm{I}}{\beta}=T_{0}^{\prime}$ and $T^{\prime}=T_{0}^{\prime}+t_{v}$, we then have the simple equation

$$
\frac{T^{\prime}}{T_{0}^{\prime}}=\frac{p}{p_{0}} \quad(v=\text { constant })
$$

The quantity $T^{\prime}$ may evidently be regarded as the temperature measured, not from the ice point but from $-T_{0}^{\prime}$. It is called the "absolute temperature" by the scale of the thermometer in question.

The definition contained in equation (5) is analogous to that of the absolute thermodynamic temperature $\theta_{0}$ given by equation (3), and is therefore incomplete until the further condition is added that the temperatures of the ice and steam points shall differ by $100^{\circ}$. The value of $T_{0}^{\prime}$ is then always approximately 273 .

The centigrade temperature on the constant-volume scale may now, when convenient, be defined by the equation

$$
t_{v}=T^{\prime}-T_{0}^{\prime}
$$

which is analogous to equation (4) and, under the condition that the ice and steam points shall be $100^{\circ}$ apart, equivalent to equation (I), the original definition of $t_{v}$.

The coefficient of expansion, which is also always nearly $\frac{I}{273}$, is defined by the equation

$$
a=\frac{v_{100}-v_{0}}{\mathrm{IOO} v_{0}}
$$

If we let $\frac{\mathrm{I}}{a}=T_{0}$ and $T=T_{0}+t_{p}$, equation (2) gives us

$$
\frac{T}{T_{0}}=\frac{v}{v_{0}} \quad(p=\text { constant })
$$

The quantity $T$ may be regarded as the temperature measured on the constant-pressure scale from $-T_{0}$, and it is known as the "abso$23835-07-6$ 
lute temperature" by the constant-pressure scale. Here again, to complete the definition we add the condition that the ice and steam points shall be $100^{\circ}$ apart, and the value of $T_{0}$ is then always approximately 273 .

The centigrade temperature by the constant-pressure scale may now be considered as defined by the equation

$$
t_{p}=T-T_{0}
$$

which is analogons to equations (4) and (6) and equivalent to equation (2), the original definition of $t_{p}$.

Let $T^{\prime}, T$, and $\theta$ be the numerical values of a given temperature on the three absolute scales. Then it may readily be shown by elementary thermodynamics that if the thermometer is filled with an ideal gas,

$$
\frac{T^{\prime}}{T_{0}^{\prime}}=\frac{T}{T_{0}}=\frac{\theta}{\theta_{0}}
$$

or that the three scales are identical. The ideal gas is defined as one which follows Boyle's law and in which a free expansion, with no external work, would canse no change in the temperature. These conditions may be written in the form

$$
\begin{gathered}
(p v)_{t}=\text { constant } \\
\lambda=0 .
\end{gathered}
$$

No real gas satisfies these conditions exactly, but all the connmon thermometric gases, as they are used in gas thermometers, do satisfy them approximately. Hence it is that the ordinary gas scales and the thermodynamic scale are all approximately the same, and the problem of finding the mutual relations of the various scales is reduced to the investigation of the departures of the actual gases from the icleal state and the computation of corrections for the departures.

Stated in this way the problem looks simple, but two difficulties arise at once: first, the variations of $p u$ at constant temperature are small and are not known with sufficient completeness; and second, the quantity $\lambda$, which may be defined as the amount of heat that would have to be added to a unit mass of gas during a free expansion 
in which its volume increased by unity to keep the temperature from changing, is so small that it has not even been observed, still less measured. The difficulty of getting the experimental data necessitates a different mode of attacking the problem.

When Joule's experiments on free expansion had failed to determine the value of $\lambda$ further than to show that it was very small, Lord Kelvin devised the well known porous-plug experiment which he then carried out in coöperation with Joule. In this experiment a single quantity is determined which depends on the departure of the gas from both of the conditions of ideality at once. If these departures could be determined separately the theory of the constantvolume thermometer would be quite simple. As it is, it is impossible to give a thoroughly satisfactory thermodynamic theory of the constant-volume thermometer on the basis of existing data, but a knowledge of the Joule-Thomson effect makes it possible to give the theory of the constant-pressure thermometer.

\section{THEORY OF THE POROUS-PLUG EXPERIMENT.}

In the porous-plug experiment the gas is forced to flow steadily through a porous plug. The plug is so insulated that no heat can enter or leave it by conduction. The pressure and temperature of the gas are observed on both sides of the plug. It was found by Joule and Lord Kelven that hydrogen became warmer, while nitrogen, air, oxygen, and carbonic acid became colder in traversing the plug.

Let $p_{1}, v_{1}, \epsilon_{1}$ represent the pressure, the specific volume, and the specific internal energy of the gas on the high-pressure side of the plug, and let $p_{2}, v_{2}, \epsilon_{2}$ be the corresponding values on the low-pressure side. Since the gas enters the plug uniformly at the constant pressure $p_{1}$, the work done on it by the pump is $p_{1} v_{1}$ per gram. The plug having sufficient resistance, the gas issues in a steady stream at the lower pressure $p_{2}$, and in issuing from the plug does a quantity of work $p_{2} v_{2}$ per gram in pushing forward the gas ahead of it. The total work done on one gram of the gas is therefore $p_{1} v_{1}-p_{2} v_{2}$. If no heat enters or leaves the gas by conduction, and if the flow is slow enough that the change in kinetic energy is negligible, the work done on the gas must be equal to the increase of its internal energy, so that we have 


$$
\epsilon_{2}-\epsilon_{1}=p_{1} v_{1}-p_{2} v_{2}
$$

This equation is rigorously true under the conditions imposed, no matter what the pressures $p_{1}$ and $p_{2}$ are. If the fall of pressure be infinitesimal, equation (I 2 ) reduces to

$$
\Delta \epsilon=-\Delta(p v)=-p \Delta v-v \Delta p
$$

In Fig. I let the initial state of the unit mass of gas be represented by the point $A$ with the coordinates $p, v, t$, the temperature $t$ being measured on any convenient scale.

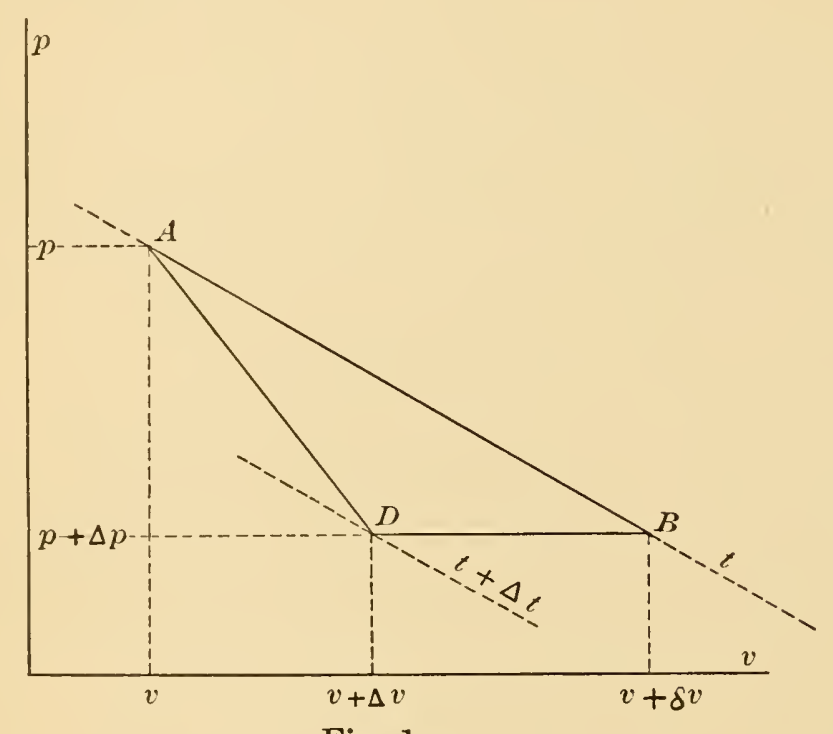

Fig. 1.

Let the state of the gas after passing the plug be represented by $D$ with the coordinates $p+\Delta p, v+\Delta v, t+\Delta t ; \Delta p$ and $\Delta t$ are negative while $\Delta v$ is positive. Let the point $B$, with the coordinates $p+\Delta p, v+\delta v, t$, represent the state the gas would have been in if, during the same fall of pressure, its temperature had been kept constant.

After the gas, in passing through the porous plug, has reached the state $D$, let it be heated at constant pressure till it attains the state $B$. Then the excess of its internal energy at $B$ over that at $A$ is

$$
\delta \epsilon=\Delta \epsilon+Q+W
$$

where $\Delta \epsilon$ is the excess of the internal energy at $D$ over that at $A$, and $Q$ and $W$ are, respectively, the heat added to the gas and the work done on it during the isopiestic change $D B$ which brings it back to its original temperature $t$. 
Let $\mu$ be the limiting value of the ratio of the fall of temperature to the fall of pressure, i. e., $\mu=\Delta t i \Delta p$. Then we have $\Delta t=\mu \Delta p$ and

$$
Q=-\mu C_{p} \Delta p
$$

where $C_{p}$ is the specific heat at constant r,ressure, in ergs. The work done on the gas is

$$
W=-p(\delta v-\Delta v)
$$

and furthermore, since the change of volume from $v+\Delta v$ to $v+\delta v$ is caused by heating at constant pressure over an interval $-\Delta t$, we have

$$
\begin{aligned}
\delta v-\Delta v & =(-\Delta t)\left(\frac{\partial v}{\partial t}\right)_{p} \\
& =-\mu\left(\frac{\partial v}{\partial t}\right)_{p} \Delta p
\end{aligned}
$$

whence

$$
\Delta v=\delta v+\mu\left(\frac{\partial v}{\partial t}\right)_{p} \Delta p
$$

Substituting in equation (14) the values given by equations (13) to (I8), we have

$$
\delta \epsilon=-p \delta v-v \Delta p-\mu C_{p} \Delta p
$$

and since the differentials in this equation now all relate to the isothermal change $A B$, it may be written

$$
\left(\frac{\partial \epsilon}{\partial v}\right)_{t}=-p-\left(v+\mu C_{p}\right)\left(\frac{\partial p}{\partial v}\right)_{t}
$$

Now it is known that in any reversible change of state of a system with only two degrees of freedom and acted on by no external forces except a uniform normal pressure,

$$
\delta \epsilon=\theta \delta \eta-p \delta v
$$

where $\eta$ is the entropy and $\theta$ the thermodynamic temperature. Equation (2O) is merely a statement of the two laws of thermodynamics for such a system. If the process is isothermal, equation (20) may be written 


$$
\left(\frac{\partial \epsilon}{\partial v}\right)_{\theta}=\theta\left(\frac{\partial \eta}{\partial v}\right)_{\theta}-p
$$

To give this a physical meaning the entropy $\eta$ must be eliminated, as follows: Substracting $\delta(\theta \eta)$ from equation (20) gives

$$
\delta(\epsilon-\theta \eta)=-\eta \delta \theta-p \delta v
$$

and since the first member is a complete differential the second is also, so that

$$
\left(\frac{\partial \eta}{\partial v}\right)_{\theta}=\left(\frac{\partial p}{\partial \theta}\right)_{v}
$$

Using this relation and noting that if $\theta$ is constant, $t$ also is constant, we may put equation (2I) into the form

$$
\left(\frac{\partial \epsilon}{\partial v}\right)_{t}=\theta\left(\frac{\partial p}{\partial \theta}\right)_{v}-p
$$

whence by comparison with equation (A) we have

$$
\theta\left(\frac{\partial p}{\partial \theta}\right)_{v}=-\left(v+\mu C_{p}\right)\left(\frac{\partial p}{\partial v}\right)_{t}
$$

an equation which may serve as the starting point of the theory of the gas thermometer when treated by the use of the JouleThomson effect.

This equation (B) is rigorously true, having been deduced solely from the two laws of thermodynamics with no further hypotheses. The quantity $\mu C_{p}$ evidently has the dimensions of a volume; it is therefore independent of the scale of temperature, provided that the same scale be used for $\mu$ as for $C_{p}$. In applying the equation to the practical question of finding the corrections of the gas thermometer, one further assumption must be made. The quantity $\mu$ can not be measured directly since it is defined as the ratio of two infinitesimals. But the experiments appear to have shown that the ratio of the change of temperature to the difference of pressure on the two sides of the plug is, for small pressures at all events, constant and independent of both the fall of pressure and the absolute value of the pressure. We assume that this constant ratio found at any given mean temperature is the same as the true value of $\mu$, and take the observed values of the ratio as values of $\mu$ for use in the equations. 
3. THEORY OF THE CONSTANT-VOLUME THERMOMETER.

If in equation $(\mathrm{B}), v\left(\frac{\partial p}{\partial v}\right)_{t}$ be replaced by its equivalent $\left[\frac{\partial}{\partial v}(p v)\right]_{t}-p$, the equation takes the form

$$
\theta\left(\frac{\partial p}{\partial \theta}\right)_{v}=p-\left[\frac{\partial}{\partial v}(p v)\right]_{t}-\mu C_{p}\left(\frac{\partial p}{\partial v}\right)_{t}
$$

Inverting, multiplying by $d p$, and integrating at constant volume between any two temperatures, $\theta_{0}$ and $\theta$, at which the pressures at the constant volume $v=\phi$ are $p_{0}$ and $p$, gives

$$
\log \frac{\theta}{\theta_{0}}=\int_{p_{0}}^{p} \frac{d p}{\left[\frac{\partial}{\partial v}(p v)\right]_{t}-\mu C_{p}\left(\frac{\partial p}{\partial v}\right)_{t}} \quad(v=\phi)
$$

If $T^{\prime}$ be the absolute temperature as measured by a constantvolume thermometer filled with the gas in question,

$$
p=p_{0} \frac{T^{\prime}}{T_{0}^{\prime}}
$$

by definition, and upon eliminating $p$ from equation (26) we have

$$
\log \frac{\theta}{\theta_{0}}=\int_{T_{T^{\prime}}}^{T^{\prime}-\frac{T_{0}^{\prime}}{p_{0}}\left[\frac{\partial}{\partial v}(p v)\right]_{T^{\prime}}-\frac{T_{0}^{\prime}}{p_{0}} \mu C_{\eta}\left(\frac{\partial p}{\partial v}\right)_{T^{\prime}}}
$$

The integration of this equation demands not only a knowledge of the value of $\mu C_{p}$ at all temperatures between $\theta_{0}$ and $\theta$, but also a complete knowledge of the isothermal compressibility of the gas within the same limits.

A slightly different method of integration may be adopted. Divide equation (25) through by $\theta^{2}$, rearrange, substitute

$$
\frac{\mathrm{I}}{\theta} \frac{\partial p}{\partial \theta}-\frac{p}{\theta^{2}}=\frac{\partial}{\partial \theta}\left(\frac{p}{\theta}\right)
$$

and integrate between $\theta_{0}$ and $\theta$; the result is

$$
\underline{p}-\frac{p_{0}}{\theta_{0}}=-\int_{\theta_{0}}^{\theta} \frac{I}{\theta^{2}}\left[\frac{\partial}{\partial v}(p v)+\mu C_{p} \frac{\partial p}{\partial v}\right]_{\theta} d \theta
$$


This equation may serve the same purpose as (C), but the data on compressibility and on the values of $\mu C_{p}$ must be given, not as before in terms of the constant-volume gas scale $T^{\prime}$, but in terms of the thermodynamic scale $\theta$ which is to be established. This point is, however, of little importance, for since the whole second member is obviously only a small correction term, no large error will be introduced by identifying $T^{\prime}$ with $\theta$ in computing this correction, and at the worst, a result may be obtained by successive approximations.

The fundamental difficulty is the necessity of having the information about the departure of the gas from Boyle's law, in addition to the specific heat, the Joule-Thomson effect, and the coefficient of pressure which determines $T_{0}{ }^{\prime}$. This difficulty is inherent in the theory of the constant-volume thermometer, so far as based on the Joule-Thomson effect. Various treatments of the subject have been given ${ }^{3}$, differing in substance only in the different methods of handling the terms $\frac{\partial}{\partial v}(p v)$ and $\frac{\partial p}{\partial v}$. From the imperfect experimental knowledge we have of the general form of the equation of state

$$
f(p, v, t)=0
$$

particular equations are set up which shall give, in various ways, the values of the correction terms in equations (C) and (D) with sufficient accuracy for the purpose in hand. The corrections to be determined are small and the final results are doubtless nearly correct, but all hypotheses regarding the compressibility are dispensed with in the theory of the constant-pressure thermometer. This superior simplicity in the theory of the constant-pressure thermometer is not due to any superiority in the constant-pressure method itself, but to the nature of the data available. The results of the porouspling experiment are, in fact, not well adapted for use in finding the corrections of the constant-volume scale. If the freeexpansion experiments of Gay-Lussac and Joule could be carried out with sufficient accuracy, they would give the values of the quantity $\lambda$, defined above as the amonnt of energy that would have

${ }^{3}$ Rose-Innes Phil. Mag. (5) 4.), p. 227; IS98; 50, p. 25I; I900; (6) 2, p. I 30; 1901: Callendar, Phil. Mag. (6) 5, 1). 4S; 1903. 
to be added to each gram of gas in an isothermal expansion in which the specific volume increased by unity and the gas did no external work. In such an expansion the internal energy of the gas would increase simply by the amount $\lambda$, and we should have the equation

$$
\left(\frac{\partial \epsilon}{\partial v}\right)_{t}=\lambda
$$

Comparison of this equation with (24) gives

$$
\lambda=\theta\left(\begin{array}{l}
\partial p \\
\partial \theta
\end{array}\right)_{v}-p
$$

which may easily be put into either of the two forms

$$
\begin{gathered}
\log \frac{\theta}{\theta_{0}}=\int_{T_{\mathrm{c}^{\prime}}}^{T^{\prime}} \frac{d T^{\prime}}{T^{\prime}+\frac{T_{0}^{\prime} \lambda}{p_{0}}} \\
\frac{p}{\theta}-\frac{p_{0}}{\theta_{0}}=\int_{\theta_{0}}^{\theta^{\theta}} \frac{\lambda d \theta}{\theta^{2}}
\end{gathered}
$$

These equations correspond to equations (C) and (D), but do not, for integration, necessitate any information regarding the departure of the gas from Boyle's law.

If the manner in which the gas departs from Boyle's law is known, it is a simple matter to find the relation of the constantvolume and constant-pressure scales. Hence if the relation of either to the thermodynamic scale is known, the relation of the other can be found.

\section{THEORY OF THE CONSTANT-PRESSURE THERMOMETER.}

Returning to equation (B), namely,

$$
\theta\left(\frac{\partial p}{\partial \theta}\right)_{v}=-\left(v+\mu C_{p}\right)\left(\frac{\partial p}{\partial v}\right)_{t}
$$

let us make use of the relation

$$
\left(\frac{\partial p}{\partial t}\right)_{v}\left(\frac{\partial t}{\partial v}\right)_{p}\left(\frac{\partial v}{\partial p}\right)_{t}=-\mathrm{I}
$$


which is rigorously true, whatever be the scale in which $t$ is measured. If $t=\theta$ and equation (B) be inultiplied by $\left(\frac{\partial v}{\partial \theta}\right)_{p} /\left(\frac{\partial p}{\partial \theta}\right)_{r}$, the result is

$$
\theta\left(\frac{\partial v}{\partial \theta}\right)_{p}=v+\mu C_{p}
$$

which is the form in which the fundamental equation of the porousplug experiment is most frequently given. In its integral form the equation is

$$
\log \frac{\theta}{\theta_{0}}=\int_{v_{0}}^{v} \frac{d v}{v+\mu C_{p}}
$$

If the absolute temperature by the constant-pressure thermometer be defined as usual by the equation

$$
\frac{v}{v_{0}}=\frac{T}{T_{0}}
$$

equation (35) takes the form

$$
\log \frac{\theta}{\theta_{0}}=\int_{T_{0}}^{T} \frac{d T}{T+\frac{T_{0} C_{p^{\prime}}}{v_{0}}}
$$

By a slightly different method of treatment, similar to one already used in the last section, equation (E) may also be thrown into the form

$$
\frac{v}{\theta}-\frac{v_{0}}{\theta_{0}}=\int_{\theta_{0}}^{\theta} \frac{\mu C_{p} d \theta}{\theta^{2}}
$$

Equations (F) and $(\mathrm{G})$ play the same part in the theory of the constant-pressure thermometer as equations (32) and (33) would play in that of the constant-volume thermometer, if we had experimental data on $\lambda$ such as we actually have for $\mu C_{p}$. If they be compared with equations (C) and (D), it is obvious that the theory of the constant-pressure thermometer, in so far as it is based on the JouleThomson effect, is decidedly simpler than that of the constant-volume thermometer, and especially that it does not necessitate making any hypotheses to help ont the imperfection of the experimental data on compressibility. 'The theory of the constant-pressure thermometer 
has thus one less weak point than that of the constant-volume thermometer.

It may be remarked that if the pressure of the gas were infinitesimal so that $v_{0}$ became infinite, while $\mu C_{p}$ remained finite, the correction term in $(\mathrm{F})$ would vanish and the integration would give

$$
\frac{\theta}{\theta_{0}}=\frac{T}{T_{0}}
$$

If, therefore, it is possible to find by extrapolation, the limit of the rate of thermal expansion of a gas as its density approaches zero, the thermodynamic scale is established directly and with no further knowledge of the value of $\mu C_{p}$ than that it does not become infinite. By means of equation (36), equation $(G)$ may be put in the form

$$
\frac{T}{T_{0}}-\frac{\theta}{\theta_{0}}=\frac{\theta}{v_{0}} \int_{\theta_{0}}^{\theta} \frac{\mu C_{p} d \theta}{\theta^{2}}
$$

from which we see again that if the gas is rarified so that $p$ approaches zero, i. e., $v_{0}=\infty$, while $\mu C_{p}$ remains finite, the constantpressure scale approaches identity with the thermodynamic scale.

Now $\mu$ has already, in accordance with the indications of experiment, been assumed to be sensibly independent of the pressure, so long as the pressure is small; and furthermore, it is known that $C_{p}$ varies only very slightly with the pressure. Hence for any given temperature $\theta$, the value of the correction term

$$
\frac{\theta}{v_{0}} \int_{\theta_{0}}^{\theta} \frac{\mu C_{p} d \theta}{\theta^{2}}
$$

is nearly proportional to $\frac{\mathrm{I}}{v_{0}}$. But since the gas follows Boyle's law approximately, $\frac{I}{v_{0}}$ is nearly proportional to the constant pressure $\pi$ to which the gas in the thermometer is subject, so that for any given temperature we have

$$
\frac{T}{T_{0}}-\frac{\theta}{\theta_{0}}=A \pi
$$


where $A$ is nearly constant. If we write this in the form

$$
\frac{T-T_{0}}{T_{0}}-\frac{\theta-\theta_{0}}{\theta_{0}}=A \pi
$$

substitute $T-T_{0}=t_{p}$ and $\theta-\theta_{0}=t_{\theta}$, and note that $T_{0}$ and $\theta_{0}$ are both approximately 273 , we get the equation

$$
t_{p}-t_{\theta}=273 A \pi
$$

We have therefore the important result that the difference between the numerical values of any given temperature on the centigrade constant-pressure scale and on the centigrade thermodynamic scale, i. e., the thermodynamic correction of the centigrade constantpressure scale at the given temperature, is very nearly proportional to the constant pressure $\pi$ at which the gas is kept.

By introducing the approximation $p v=R \theta$ and treating equation (D) as equation $(G)$ has just been treated, we may reach a similar result for the constant-volume thermometer, namely, that the thermodynamic corrections of the centigrade constant-volume scale are approximately proportional to the initial pressure $p_{0}$ at the ice point.

These two propositions are very useful, for they enable us, after finding the corrections for any gas thermometer of either type, to compute at once and with quite sufficient exactness the corrections for the same gas at any other pressure.

\section{NUMERICAL DATA ON $v_{0}, C_{p}$, AND $T_{0}$.}

The numerical data needed for finding the relations of the constant-pressure scales of air, nitrogen, and hydrogen to the thermodynamic scale will next be considered. These data, some of the latest of which do not appear to have been used for this purpose, will then be ntilized in computing the thermodynamic temperature of the ice point, and in computing corrections at varions temperatures.

In order to make practical use of equation $(\mathrm{F})$ we nust know the values of the constants $\tau_{0}$ and $T_{0}$, and the value of $\mu C_{p}$ as a function of $T$ throughout the range of temperature $T_{0}$ to $T$. The ice point will be taken for the initial temperature $T_{0}$; its numerical value on the constant-pressure scale is the reciprocal of the mean coefficient 
of expansion between the ice and stean points. The specific volume is to be taken at the pressure for which the coefficient of expansion is determined. The specific heat $C_{p}$ is nearly constant, and since it occurs only in a small correction term, it is sufficient to treat it as a constant and use its mean value for the given interval. The JonleThomson effect will be considered separately and at length.

(a) The Specific Volumes. - Of the four quantities $v_{0}, T_{0}, C_{p}$, and $\mu$, which appear in the correction term, the specific volume and the absolute temperature of the ice point are the most exactly known. The temperature of the ice point, which also appears as the lower limit of the integration, nust be known with the highest attainable exactness, but in the currection term the uncertainties of $v_{0}$ and $T_{0}$ are of much less importance than those of $C_{p}$ and especially of $\mu$. The best data available have, however, been used for the specific volume $v_{0}$, but it has not been thought necessary to refer to all the original papers, the figures having been taken from an article by D. Berthelot ${ }^{4}$, which contains a very complete and useful discussion of the densities and compressibilities of a number of the more permanent gases.

The data needed are the density $\rho_{0}$ at the ice point and under a pressure of one atmosphere, and the mean value of the coefficient $K$ in the equation

$$
p_{2} v_{2}=p_{1} v_{1}\left[\mathrm{I}+K\left(p_{2}-p_{1}\right)\right]
$$

within the small range of the reduction here needed. In Table I are given for air, nitrogen, and hydrogen the values of: (I) the density according to the experiments of Leduc, Morley, and Rayleigh; (2) the coefficient $K$ according to the experiments of Chappuis, Leduc and Sacerdote, and Rayliegh (the value for air is taken from Regnault); (3) the pressures $\pi$ for which the specific volumes at the ice point are to be found; (4) the specific volumes for these pressures as computed by means of equation (39) from the values of $\rho_{0}$ and $K$ just given. It seems highly improbable that any of these values of $v_{0}$ is in error by as much as I/IOOO, and such an error is of small importance compared with the uncertainties in the values of $C_{p}$ and $\mu$. The values of $K$ assume one atmosphere as the unit of pressure.

${ }^{4}$ Zeitschr. E1. Chem., 10, p. 62I; I904. 


\section{TABLE I.}

Specific Volumes.

\begin{tabular}{|c|c|c|c|c|}
\hline & $\rho_{0}$ & $K_{0}$ & $\pi$ & $v_{0}$ \\
\hline Air & 0.00129278 & -0.00191 & $1000.8 \mathrm{~mm}$ & 587.015 \\
\hline Nitrogen & 0.00125034 & -0.000559 & 1001.9 & 606.626 \\
\hline Hydrogen & 0.000089846 & +0.000772 & 1000.5 & 8461.24 \\
\hline
\end{tabular}

(b) The Specific Heats. - A collection of data on the specific heats may be found on page 406 of the third edition of Landolt and Börnstein's tables.

For air, the values given by Regnault, Witkowski, and Lussana for various temperatures between $-20^{\circ}$ and $+200^{\circ}$ lie between 0.2370 and 0.2378 with no indication of systematic variation with the temperature. E. Wiedemann obtained the value 0.2389 , and Holborn and Anstin ${ }^{5}$ have recently obtained, for the mean value between $20^{\circ}$ and $440^{\circ}, 0.2366$ observed directly, and 0.2377 computed from measurements on oxygen and nitrogen separately. These values are all for a pressure of one atmosphere. The variation of the specific heat with pressure was investigated by Lussana ${ }^{6}$, who found for air

$$
C_{p}=0.23707+0.001498(p-\mathrm{I})
$$

where $p$ is given in atmospheres. Lussana's value will be used. The range of temperature in lis experiments was from $94^{\circ}$ to the nean temperature of the calorimeter, which was $28^{\circ}$. Taking for the mechanical equivalent of heat at $28^{\circ}$, the value $J=4.173 \times \mathrm{IO}^{7} \mathrm{ergs}$, we get for the specific heat of air at the constant pressure of Ioor $11 m$

$$
C_{p}=9.913 \times \mathrm{IO}^{6} \text { ergs. }
$$

For nitrogen, the only experimental data available are those recently given by Holborn and Austin. ${ }^{5}$ Their observations may be represented by the equation

$$
\left[C_{1}\right]_{0}^{\mathrm{t}}=0.2322+0.00002 \mathrm{I} 55 t
$$


where $\left[C_{p}\right]_{0}^{t}$ is the mean specific heat, at atmospheric pressure, between $0^{\circ}$ and $t^{\circ}$. Assuming that the specific heat varies with the pressure in the same way that Lussana found for air, the value becomes, for a pressure of $1002 \mathrm{~mm}$,

$$
\left[C_{p}\right]_{0}^{\mathrm{t}}=0.2327+0.00002 \mathrm{I} 6 t
$$

The calorimeter temperature was $18^{\circ}$, for which we may set $J=4.182 \times 10^{7}$ and so get, finally, the formula

$$
\left[C_{p}\right]_{0}^{\mathrm{t}}=(9.73 \mathrm{I}+0.000903 t) \times 10^{6} \text { ergs. }
$$

For hydrogen, the values of the specific heat at a pressure of one atmosphere and at various temperatures between $-30^{\circ}$ and $+200^{\circ}$, as given by Regnault, E. Wiedemann, and Lussana, fall between 3.3996 and 3.4IO with no indication of systematic variation with the temperature. Lussana's ${ }^{6}$ value will be used, namely,

$$
C_{p}=3.4025+0.0133(p-\mathrm{I})
$$

The mean temperature of the calorimeter was $28^{\circ}$ to $29^{\circ}$, so that $J$ may be given the same value as before, and the result for a pressure of $1000 \mathrm{~mm}$ is

$$
C_{p}=\mathrm{I} .42 \mathrm{I} 8 \times 1 \mathrm{IO}^{8} \text { ergs. }
$$

(c) The Coefficients of Expansion.-For the coefficient of expansion $a$, of which $T_{0}$ is the reciprocal, the most recent data by Chappuis $^{2}$ have been used. These values, together with the values of $v_{0}$, and of $\left[C_{p}\right]_{0}^{100}$ already given, are collected in Table II.

\section{TABLE II.}

Data on the Specific Volumes, Specific Heats, and Coefficients of Expansion of Air, Nitrogen, and Hydrogen.

\begin{tabular}{c|c|c|c}
\hline & Air & Nitrogen & Hydrogen \\
\cline { 2 - 3 } \cline { 4 - 4 } & 1000.778 & 1001.855 & 1000.460 \\
$a$ & 0.00367282 & 0.00367315 & 0.00366004 \\
$T_{0}$ & 272.270 & 272.246 & 273.221 \\
$v_{0}$ & 587.01 & 606.63 & 8461.2 \\
$C_{p}$ & $9.913 \times 10^{6}$ & $10.091 \times 10^{6}$ & $142.18 \times 10^{6}$ \\
\hline
\end{tabular}

'Trav. et Mém. Bur. Int., XIII, I903. 


\section{EXPERIMENTAL DATA ON THE JOULE-THOMSON EFFECT.}

There 11111st, finally, be considered the numerical data on the JonleThomson effect upon which there are available: (I) the experiments of Joule and Lord Kelvin ${ }^{8}$ on hydrogen, nitrogen, air, oxygen, and carbonic acid; (2) the experiments of E. Natanson ${ }^{9}$ on carbonic acid, which will not be used because they relate only to room temperatures; (3) the experiments of $\mathrm{Kester}^{10}$ on carbonic acid. The experimental results must be examined somewhat in detail.

There are, to start with, twenty-two experiments on air. The authors group the results about the temperatures $7^{\circ}$. I, $39^{\circ} \cdot 5$ and $92: 8$, and represent them by an equation of the form $\mu=\frac{a}{T^{2}}$. If, however, all the twenty-two values of $\mu$ be plotted separately against the temperatures, it is at once evident that the experimental errors are so large as to make the deduction of anything but a linear equation between $\mu$ and $T$ from these observations alone, a somewhat visionary refinement. Taking the values of the cooling effect in centigrade degrees for a fall of pressure at the plug of roo inches of mercury, as given in the tables, the best straight line that can be drawn to represent the results has approximately the equation

$$
\mu=0.908-0.00434^{t}
$$

where $t$ is the centigrade temperature.

Upon nitrogen, three experiments were made, one at $7^{\circ} \cdot 2$, one at $91^{\circ} \cdot 4$, and one at $92^{\circ}$, the nitrogen having been diluted with oxygen to the extent of 7.9 per cent, 2.2 per cent, and 12.5 per cent respectively, in the three cases. The method of finding the value of $\mu$ for pure nitrogen does not appear to be stated specifically, but it was doubtless by means of the mixture rule. The best that can be done with these observations, by themselves, is to determine a straight line from then1; its equation is

$$
\mu=1.068-0.00475 t
$$

The two results at the higher temperature differ by nearly 20 per cent.

\footnotetext{
${ }^{8}$ Kelvin, Math. and P'hys. Papers, 1, pp. 419-424.

${ }^{9}$ Wied. Ann., :31, p. 502; 1888.

${ }^{10}$ Pliy's. Rev., 21, p. 260; 1905.
} 
With hydrogen, seventeen experiments were nade, in some of which the hydrogen formed only a small impurity in a mass of air. The value of $\mu$ at $o^{\circ}$ is only about one-ninth as large for hydrogen as for air, so that the correction needed in getting the value for pure hydrogen is large and therefore uncertain. It seems by all means best to disregard entirely the results obtained when the amount of impurity was large. There then remain nine experiments in which the impurity in the hydrogen was less than 6.I per cent. Four of these, at a mean temperature of $6: 8$, gave values of $\mu$ from -0.075 to -0.126 with a mean of -0.099 I, while the remaining five, at a mean temperature of 90 : I, gave values from -0.098 to -0.234 with a mean of -0.1483 . The straight line thus determined has the equation

$$
\mu=-0.095 \mathrm{I}-0.00059^{t}
$$

Upon oxygen, six experiments were carried out. In three of these the percentages of nitrogen mixed with the oxygen were 54.6, 22.4, and 5 I.O, respectively, while in the others the percentages were 5.I, 3.6, and 4.0. Throwing out the first three, for obvious reasons, we have remaining one experiment at 8.7 , one at $89^{\circ} \cdot 5$, and one at $95^{\circ} \cdot 5$, of which the last two give values of $\mu$ differing by over onethird of their mean.

The seventeen values obtained by Joule and Lord Kelvin in their experiments on carbonic acid are grouped about five temperatures. When the mean values of $\mu$ are plotted against the temperature, the curve $\mu=f(t)$ has a distinct curvature with its convexity toward the origin. The gas was always somewhat impure and the results for pure carbonic acid were computed by a modification of the inixture rule. Kester's experiments, in which the values for the pure gas were found by graphical extrapolation from the results obtained with varying amounts of impurity, appear more reliable and his results will be used in what follows. They give a curve similar to that obtained by Joule and Lord Kelvin, but the values of $\mu$ are somewhat larger and the agreement of the separate experimental values is better.

From this brief summary of the available data on the JouleThomson effect it may be seen that our quantitative knowledge of this phenomenon is very imperfect. Of the three thermometric $23835-07-7$ 
gases, air, nitrogen, and liydrogen, air, the least important, is the only one upon which any extended series of observations at different temperatures has been made, and even in this case the observations leave much to be desired. Furthermore, all the observations are confined between the limits $0^{\circ}$ and $100^{\circ}$, and they are too evidently inaccurate to give any feeling of security in using an empirical equation deduced from them for the purpose of extrapolation outside these limits. The observations will therefore be examined from another point of view, and an attempt made to improve the situation with regard to the Joule-Thomson effect.

\section{APPLICATION OF THE LAW OF CORRESPONDING STATES.}

In its most general form, the law of corresponding states affirms that if for each particular substance the pressure, volume, and temperature be measured in the proper units and from the proper zero points, there exists a single surface,

$$
f(p, v, t)=0
$$

common to all substances ${ }^{11}$. In the more special form in which it was first announced by van der Waals ${ }^{1:}$, it says that if the pressures, volumes, and absolute temperatures be measured for each substance in terms of their values at the critical point, the equation of state is the sane for all substances and contains no quantities dependent on the special properties of particular substances. In this more specific form the law is not generally true, but it appears to be very approximately true within particular groups of substances. It will now be applied to the five gases for which the Joule-Thomson effect has been neasured, namely, liydrogen, nitrogen, air, oxygen, and carbonic acid. This seems justifiable a priori for air and the three diatomic gases. For carbonic acid we have the fact that its isothermals, when reduced to the proper scale, are congruent with those of air, as has been shown by Anlagat ${ }^{13}$, though they are not represented by van der Waals's equation as shown by Ravean ${ }^{14}$ from Amagat's,

${ }^{11}$ See K. Meyer, Zeitsclır. Phys. Chem1., 32, p. I ; 1900.

12 Über die Continutät des gasförnigen und flüssigen Zustandes.

${ }^{13}$ Journ. de Phys. (3), 6, p. 5; 1897.

${ }^{14}$ Journ. de Phys. (3), 6, p. 432; 1897. 
experimental data. It will be assumed, then, that for these five gases "corresponding" states are states in which the pressures, volumes, and temperatures are the same fractions of the critical pressures, volumes, and temperatures, the temperatures being counted from $-273^{\circ}$, and that the properties of the gases are expressible in terms of these "reduced" units by a single equation of state.

It follows that if the gases be taken in corresponding states and subjected to the porous-plug experiment, a fall of pressure at the plug by a given fraction of the critical pressure will cause a cooling effect which is for all the gases the same fraction of the critical temperature. Now experiment has shown that for the small pressures used in gas thermometry, the thermal effect per unit fall of pressure at the plug is sensibly independent not only of the fall of pressure but of the initial value of the pressure, and consequently of the initial specific volume. Hence for present purposes, so long as the pressures are only a few atmospheres, the gases may be considered to be in corresponding states if only they are at the same reduced temperature, i. e., each at the same fraction or multiple of its critical temperature measured from $-273^{\circ} \mathrm{C}$. The proposition under consideration may then be stated as follows: "At equal reduced temperatures, the reduced Joule-Thomson effect is the same," meaning by the "reduced Joule-Thomson effect" the ratio of the change in the reduced temperature to the fall in the reduced pressure at the plug.

But the Joule-Thomson effect changes with the temperature; the cooling effect decreases as the mean temperature of the experiment rises, changing finally into a heating effect, for hydrogen at all events; and it is the nature of this change, or the form of the relation $\mu=f(\tau)^{15}$, as well as the absolute value of $\mu$, that is needed in integrating the equations of the constant-pressure thermometer. So far as the law of corresponding states is valid, this function must be the same in general form for all the gases, and if $\mu$ and $\tau$ are expressed in reduced units by $\mu^{\prime}$ and $\tau^{\prime}$ there must be an equation

$$
\mu^{\prime}=f\left(\tau^{\prime}\right)
$$

which is the same for all the gases, not merely in general form but also as regards numerical values.

${ }^{15}$ The symbol $\tau$ is used here to signify $273+t$. 
To put this in practical shape let us write

$$
\mu^{\prime}=\mu \frac{p_{c}}{\tau_{c}}
$$

where $p_{c}$ and $\tau_{c}$ are the critical pressure and critical temperature of the gas in question, and $\mu$ is the cooling observed, in centigrade degrees, when the pressures on the two sides of the porous plug differ by Ioo inclies of mercury. Reduced to practical terms our proposition then is that if all the values of $\mu^{\prime}$ for all five gases be plotted as ordinates against the reduced temperatures $\tau^{\prime}=\frac{\tau}{\tau_{c}}$ as abscissas, the points will all lie along a single smooth curve. If it is found that this is the case, within the apparent limits of error of the observations, we shall be justified in using this curve found from all the experiments as a more probable representation of the actual facts than conld be obtained from the few observations available for each particular gas. If the curve can be represented by any empirical equation, then by changing back from reduced to ordinary units, a separate equation

$$
\mu=f(\tau)
$$

may be found for each gas, the same in general form for all but with different values of the constants.

In Table III are given, for the five gases mentioned above: (I) the critical temperatures measured from $-273^{\circ} \mathrm{C}$; (2) the critical pres-

TABLE III.

Critical Constants.

\begin{tabular}{l|c|c}
\hline \multicolumn{1}{c|}{ Gas } & $\tau_{c}$ & $p_{c}$ \\
\hline Carbonic acid & 304.4 & 72.9 \\
Oxygen & 154.2 & 50.8 \\
Air & 133 & 39.3 \\
Nitrogen & 128 & 33.6 \\
Hyolrogen & 32 & 19.4 \\
\hline
\end{tabular}

sures in atmosplieres. 'These figures are taken from Landolt and Börnstein (3l. ed., pp I 82-186) and from Mathias (Le Point Critique 
des Corps Purs, Paris, I 904). The value $p_{c}=\mathrm{I} 3$ atmospheres is also given for hydrogen, and if the equation of Clausius be assumed, it agrees better with Chappuis' values of the compressibility of hydrogen than does the higher value, $p_{c}=x 9.4$ atmospheres.

These values of the critical constants have been used in computing the reduced values of $\mu$, namely,

$$
\mu^{\prime}=\mu \frac{p_{c}}{\tau_{c}}
$$

which, with the reduced temperatures, are shown in Table IV. In this table are giren: ( $\mathrm{I}$ ) the observed cooling effect in centigrade degrees for a fall of pressure of roo inches of mercury, according to Kester for carbonic acid, and according to Joule and Kelvin for the other gases; (2) the centigrade temperatures at which the values were observed; (3) the number of separate experiments on which each value is based; (4) the reduced temperatures of experiment, i. e., $\tau^{\prime}=\tau \tau_{c}$; (5) the reduced cooling effects, $\mu^{\prime}=\mu p_{c} \tau_{c}$; (6) the values of $\mu^{\prime}$ computed by the equation

$$
\mu_{1}^{\prime}=\frac{0.555}{\tau^{\prime}-0.42}-0.008\left(\tau^{\prime}-0.42\right)-0.054
$$

( 7 ) the values of $\mu^{\prime}-\mu_{1}^{\prime}$; (8) the values of $\mu^{\prime}$ computed by the equation

$$
\mu_{2}^{\prime}=\frac{0.7}{\tau^{\prime}-0.32}-0.123
$$

(9) the values of $\mu^{\prime}-\mu_{2}^{\prime}$.

On Plate I the separate values of $\mu^{\prime}$ are shown together with the curve plotted from equation (40). The values for hydrogen were obtained by using $p_{c}=\mathrm{I} 9.4 \mathrm{~atm}$. The point at $\tau^{\prime}=6.02$ is the inversion point of hydrogen found by Olszewski ${ }^{16}$ at $-80^{\circ} \cdot 5 \mathrm{C}$. Since the excess of pressure in Olszewski's experiments was over roo atmospheres, it could hardly have been expected that this point would fall in so well with the others ${ }^{17}$.

${ }_{16}$ Phi1. Mag. (6), 3, p. 535; I902; Ann. Phys., 7, p. 818, 1902.

${ }^{17}$ See A. W. Porter, Phil. Mag. (6), 11, p. 554; 1906. 
TABLE IV.

Joule-Thomson Effect in Reduced Units.

\begin{tabular}{|c|c|c|c|c|c|c|c|c|c|}
\hline & I & 2 & 3 & 4 & 5 & 6 & 7 & 8 & 9 \\
\hline Gas & $\begin{array}{c}\begin{array}{c}\text { Cooling } \\
\text { ob- } \\
\text { served }\end{array} \\
\mu\end{array}$ & $\begin{array}{c}\text { Temp. } \\
\text { of Expt. } \\
t^{\circ} \mathrm{C}\end{array}$ & $\begin{array}{l}\text { Num- } \\
\text { ber of } \\
\text { Ex- } \\
\text { pts. }\end{array}$ & $\begin{array}{c}\text { Re- } \\
\text { duced } \\
\text { temper- } \\
\text { ature } \\
\tau^{\prime}\end{array}$ & $\begin{array}{c}\begin{array}{c}\text { Re- } \\
\text { duced } \\
\text { cooling }\end{array} \\
\mu^{\prime}\end{array}$ & $\mu_{1}^{\prime}$ & $\mu^{\prime}-\mu_{1}^{\prime}$ & $\mu_{2}^{\prime}$ & $\mu^{\prime}-\mu_{2}^{\prime}$ \\
\hline $\mathrm{CO}_{2}$ & 4.82 & -0.4 & 8 & 0.896 & 1.154 & 1.081 & +0.073 & 1.092 & +0.062 \\
\hline “ & 3.92 & 20.4 & 11 & 0.964 & 0.986 & 0.962 & +0.024 & 0.964 & +0.022 \\
\hline " & 3.47 & 39.5 & 3 & 1.027 & 0.830 & 0.855 & -0.025 & 0.867 & -0.037 \\
\hline " & 3.17 & 59.5 & 3 & 1.092 & 0.759 & 0.767 & -0.008 & 0.784 & -0.025 \\
\hline " & 2.90 & 79.5 & 1 & 1.158 & 0.695 & 0.692 & +0.003 & 0.712 & -0.017 \\
\hline “ & 2.63 & 96.5 & 2 & 1.214 & 0.629 & 0.639 & -0.010 & 0.660 & -0.031 \\
\hline $\mathrm{O}_{2}$ & 1.07 & 8.7 & 1 & 1.827 & 0.352 & 0.329 & +0.023 & 0.341 & +0.011 \\
\hline " & 0.80 & 89.5 & 1 & 2.351 & 0.264 & 0.218 & +0.046 & 0.220 & +0.044 \\
\hline " & 0.57 & 95.5 & 1 & 2.390 & 0.188 & 0.212 & -0.024 & 0.215 & -0.027 \\
\hline Air & 0.88 & 7.1 & 8 & 2.106 & 0.260 & 0.260 & \pm 0.000 & 0.269 & -0.009 \\
\hline “ & 0.75 & 39.5 & 8 & 2.350 & 0.222 & 0.218 & +0.004 & 0.222 & \pm 0.000 \\
\hline " & 0.51 & 92.8 & 6 & 2.750 & 0.151 & 0.165 & -0.014 & 0.165 & -0.014 \\
\hline $\mathbf{N}_{2}$ & 1.03 & 7.2 & 1 & 2.189 & 0.271 & 0.246 & +0.025 & 0.252 & +0.019 \\
\hline " & 0.58 & 91.4 & 1 & 2.847 & 0.151 & 0.155 & -0.004 & 0.154 & -0.003 \\
\hline “ & 0.69 & 92.0 & 1 & 2.852 & 0.201 & 0.155 & +0.046 & 0.154 & +0.047 \\
\hline $\mathbf{H}_{2}$ & -0.099 & 6.8 & 4 & 8.74 & -0.060 & -0.054 & -0.006 & 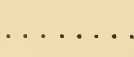 & \\
\hline$p_{c}=19.4$ & -0.148 & 90.1 & 5 & 11.34 & -0.090 & -0.091 & +0.001 & & \\
\hline  & \pm 0.000 & -80.5 & & 6.02 & \pm 0.000 & -0.001 & +0.001 & & $\ldots \ldots$ \\
\hline $\mathrm{H}_{2}$ & & & & & -0.040 & & & -0.040 & \pm 0.000 \\
\hline$p_{c}=13$ & & & & & -0.060 & & & -0.059 & +0.001 \\
\hline “ & & & & & \pm 0.000 & & & \pm 0.000 & \pm 0.000 \\
\hline
\end{tabular}

Examination of Table IV and Plate I will show that equation (40). represents the observations, well within their apparent experimental errors. Equation (4I) represents all the observations about as well as equation (40) except in the case of carbonic acid.

We have thus found that the points representing the reduced observations do lie along one smooth curve as nearly as we can tell. It therefore seems justifiable to assume that this curve, based on all the observations, gives a better representation of the facts than can be obtained from the few observations on each gas separately, and this assumption will be nagi. 
By reducing back to temperatures in centigrade degrees and expressing $\mu$ as the fall of temperature for a fall of pressure at the plug of one dyne per square centimeter, we may obtain an unreduced equation for each gas having the form

$$
\mu=\frac{a}{\tau-b}-c(\tau-b)-d
$$

if equation (40) be used, or

$$
\mu=\frac{a_{1}}{\tau-b_{1}}-d_{1}
$$

if equation (4I) be used. The constants $a, b, c, d$, or $a_{1}, b_{1}, d_{1}$, will, of course, have a different set of values for each gas. It appears at first sight that these values will be considerably affected by errors in the critical data. The coefficients of the reduced equation (40) or (4I) will be affected somewhat; but these errors are in the main eliminated by using the same critical constants in reducing back to ordinary units, and can become of importance only in the case of a wide extrapolation beyond the limits of temperature within which the experiments were made. The only large errors likely to occur are in the case of hydrogen, for which the values of $\mu$ are also small and therefore uncertain.

The values of the constants of equations (42) and (43) are shown in Table V. The values for hydrogen are based on $p_{c}=\mathrm{r} 9.4$ for the constants of equation (42), and on $p_{c}=\mathrm{r}_{3}$ for the constants of equation (43).

TABLE V.

Values of the Constants of Equations (42) and (43).

\begin{tabular}{c|c|c|c}
\hline & Air & Nitrogen & Hydrogen \\
\cline { 2 - 4 } $\log _{10} a$ & $\overline{5} .86687$ & $\overline{5} .90264$ & $\overline{6} .93706$ \\
$b$ & 55.86 & 53.76 & 13.44 \\
$\log _{10} c$ & $\overline{11.77897}$ & $\overline{11} .84702$ & $\overline{10} .08556$ \\
$\log _{10} d$ & $\overline{8} .73212$ & $\overline{8} .78353$ & $\overline{8} .42001$ \\
\hline $\log _{10} a_{1}$ & $\overline{5} .96752$ & $\overline{4} .00229$ & $\overline{5} .21057$ \\
$b_{1}$ & 42.56 & 40.96 & 10.24 \\
$\log _{10} d_{1}$ & $\overline{7} .08848$ & $\overline{7} .13989$ & $\overline{8} .95023$ \\
\hline
\end{tabular}




\section{REMARKS ON THE EQUATION $\mu=f(\tau)$.}

The general form of the reduced equation (40) was decided on by inspection of a smooth curve drawn so as to represent the observations as well as possible, and the constants were adjusted by trial. This curve has its lower asymptote inclined, and the observations can not be well reproduced by making the asymptote horizontal, if the value $p_{c}=19.4$ be adopted for hydrogen. On the other hand, the omission of the term $c(\tau-b)$ makes it impossible to represent the values for carbonic acid so well, though the simplified equation reproduces the hydrogen values a little better, on the assumption that the critical pressure of hydrogen is 13 atmospheres instead of I9.4. The values of the constants might be changed slightly, but no great improvement could be obtained. The values given in Table $\mathrm{V}$ are those that have been used in the computations in the remainder of the work.

Equations (42) and (43) are quite similar in form to those proposed by Rose-Innes ${ }^{18}$, namely,

$$
\mu=\frac{A}{T}-B
$$

and by $\mathrm{D}$. Berthelot ${ }^{19}$, namely,

$$
\mu=\frac{A}{\theta^{2}}-B
$$

and having four and three arbitrary constants, respectively, can naturally be made to fit the observations somewhat better than the simpler equations with only two constants. It is obvions, however, that unless the observations on carbonic acid are very crroneous, the curve is asymptotic to a line at about $\tau^{\prime}=0.4$ and not to the vertical axis. Jonle and Kelvin's observations on carbonic acid, which are also plotted in Plate I, though they were not considered in making np the equations, point even more strongly in the same direction. It will be noticed npon exanination of the separate points, that in deciding upon the equation of the curve, the observations on air, for which the experiments were most numerous, have

\footnotetext{
${ }^{18}$ Phil. Magr. (5), 45, p. 227; 1898.

${ }_{19}$ Trav. et Mém. Bur. Int., XIII; 1903.
} 
been given more weight than those on oxygen and nitrogen; also, that of the two observations on nitrogen at the higher temperature, the one in which the gas had the least impurity ( 2.2 per cent) lies much closer to the curve than the other in which the gas was more impure (I 2.5 per cent). It thus seems probable that if our use of the law of corresponding states is justifiable at all, the middle part of the curve has about the right position. To fit either equation (44) or equation (45) to the observations, we must either raise the iniddle part of the curve, thus spoiling the agreement for air, or else content ourselves with very rough approximation in the case of carbonic acid.

Regarding equation (45) it should be stated that M. Berthelot deduces the equation

$$
\mu M C_{p}=\frac{A}{\theta^{2}}-B
$$

where $M$ is the molecular weight, from theoretical considerations on the form of the equation of state by the aid of the law of corresponding states. If $M C_{p}$ is constant this reduces to equation (45). In the case of carbonic acid, $C_{p}$ decreases sensibly as the temperature falls; hence $\mu$ would have to increase more rapidly with falling temperature than is indicated by equation (45). In other words, if this variation of specific heat were taken into account, the carbonic acid points on Plate I would all be raised and representation of all the observations by an equation with the vertical axis as an asymptote would still be imperfect.

The main result of the last two sections is the establishment of the fact that the observations on the Joule-Thomson effect may all be represented by a single empirical equation. Equation (42) is the one that has been used in most of the following computations, though results have in some cases also been obtained by the use of equation (43) for the sake of comparison. It is assumed that this equation (42) represents the true values of the Joule-Thomson effect from about the critical temperature to some twelve times the critical temperature. The equation thus affords a reasonable means of extrapolating down to the critical temperature of hydrogen and up to twelve times the critical temperature of nitrogen or about $\mathrm{I} 200^{\circ} \mathrm{C}$. Within this range, the use of the equation is in a certain 
sense only an interpolation by an empirical formula adjusted to the observations. Outside the range $\tau^{\prime}=\mathrm{I}$ to $\tau^{\prime}=\mathrm{I} 2$, the use of the equation is in every sense an extrapolation and therefore hazardous.

\section{INTEGRATION OF THE EQUATIONS OF THE CONSTANT-PRESSURE THERMOMETER.}

The first fundamental equation for the constant-pressure thermometer is

$$
\log _{\theta_{0}} \frac{\theta}{\int_{T_{0}}}=\int_{\frac{d T}{T+\frac{T_{0} C_{p} \mu}{v_{0}}}}^{T} \quad(p=\pi)
$$

In this equation $T_{0}$ and $v_{0}$ are constants, and $C_{p}$ varies so little that it may safely be treated as a constant. The cooling effect $\mu$ has been shown to be capable of representation by an equation of the form

$$
\mu=\frac{a}{\tau-b}-c(\tau-b)-d
$$

The difference between $\tau$, or $t+273$, and $T$ is so small that we shall not commit any serious error by replacing $\tau$ by $T$ in equation (46). If this expression for $\mu$ be substituted in equation (F) the result of the integration is too complicated for convenient use in computation.

Now our first problem is the determination of the value, in centigrade degrees, of the thermodynamic temperature of the ice point, and for this purpose no serious extrapolation outside the range of the observations on $\mu$ is needed. Hence it is permissible, in the integration, to substitute for the general expression (46) any other which gives a curve nearly coincident with that given by equation. (46) between $0^{\circ}$ and $100^{\circ} \mathrm{C}$. We do not in this way throw away the work done in obtaining the more general equation, for the absolute values of $\mu$ will be based on the numerical values of the constants in the general reduced equation, i. e., on all the observations and not merely on the few for each gas. If two such simpler expressions for $\mu$ be used, one of which represents a curve much closer to that of the general equation than the other, and if the computed values of the temperature of the ice point be sensibly the 
same, it is certain that neither of them differs sensibly from the result that would have been obtained from the general expression itself.

The simplest equation is that of a straight line

$$
\mu=a-b T
$$

If this be substituted in $(\mathrm{F})$ the result of the integration is

$$
\frac{\theta}{\theta_{0}}=\left[\frac{T+A B}{T_{0}+A^{\prime} B}\right]^{v_{0} / B}
$$

where

$$
\left.\begin{array}{l}
A=a T_{0} C_{p} \\
B=v_{0}-b T_{0} C_{p}
\end{array}\right\}
$$

This equation has been used to compute the value of $\theta_{0}$, the thermodynamic temperature of the ice point, by setting $\theta=\theta_{0}+$ Ioo and $T=T_{0}+$ roo. The values of $a$ and $b$ in equation (47) which determine the constants $A$ and $B$ of equations (48) and (49) were found: first, directly from the observations, i. e., from the straight line equations given in $\S 6$; and second, by computing the values they would have for straight lines, intersecting the curves defined by equation (46) at $20^{\circ} \mathrm{C}$ and $80^{\circ} \mathrm{C}$. The values thus found for $\theta_{0}$ will be tabulated in the next section.

A second method is to use the equation

$$
\mu=\frac{a}{T^{2}}
$$

given by Joule and Lord Kelvin. This does not represent the facts at all for hydrogen; but for oxygen, nitrogen, and air between $0^{\circ}$ and $100^{\circ}$ the reduced equation

$$
\mu^{\prime}=\frac{\mathrm{I} .2 \mathrm{I} 35}{\tau^{\prime 2}}
$$

represents the observations within their experimental errors. From this equation may be obtained the values $\log _{10} \alpha=\overline{2} .33047$ for air, and $\log _{10} a=\overline{2} .34860$ for nitrogen. Using the value of $\mu$ given by equation (50) the integral of equation $(F)$ becomes

$$
\frac{\theta}{\theta_{0}}=\left[\frac{T^{3}+a T_{0} C_{p} / v_{0}}{T_{0}^{3}+a T_{0} C_{p} v_{0}}\right]^{\frac{1}{3}}
$$


which looks simpler than (48) but is not so easy to use in extended computations. The values of $\theta_{0}$ found in this way from the data on air and nitrogen will be given in the next section.

Rose-Innes's equation

$$
\mu=\frac{A}{T}-B
$$

which is a much better approximation than either $(47)$ or $(50)$ might also be used, but the result of integrating equation ( $F$ ) on this basis is nearly as complicated and unsuitable for computation as those obtained from the more general equations (42) and (43).

As a check on the foregoing methods, the general expressions have also been used in equation $(\mathrm{G})$, namely,

$$
\frac{v}{\theta}-\frac{v_{0}}{\theta_{0}}=\int_{\theta_{0}}^{\theta} \frac{\mu C_{p} d \theta}{\theta^{2}}
$$

The values of the thermodynamic temperature $\theta$ between the ice and steam points are almost certainly within $0: 3$ of $\tau$, i. e., of the ordinary centigrade temperatures by the normal scale, plus $273^{\circ}$. Since the whole second member of equation $(G)$ is merely a small correction term, no serions error will be caused by writing

$$
\mu=\frac{a}{\theta-b}-c(\theta-b)-d
$$

or

$$
\mu=\frac{a_{1}}{\theta-b_{1}}-d_{1}
$$

i. e., by iclentifying $\tau$ with $\theta$ in this correction term, and using the numerical valnes of the constants already given in Table V. The results of making these substitutions and integrating equation $(\mathrm{G})$ are

$$
\begin{aligned}
\frac{v}{\theta}-\frac{v_{0}}{\theta_{0}}=C_{1 \prime} & {\left[\left(\frac{a}{b}-c b+d\right)\left(\mathrm{I}-\frac{\mathrm{I}}{\theta}\right)+\frac{a}{\theta_{0}} \log _{\mathrm{e}} \frac{\theta_{0}(\theta-b)}{\theta\left(\theta_{0}-b\right)}\right.} \\
& \left.-c \log _{\mathrm{e}} \frac{\theta}{\theta_{0}}\right]=\phi
\end{aligned}
$$


and



Since by definition

$$
\frac{v}{v_{0}}=\frac{T}{T_{0}}
$$

it follows from (54) that

$$
\frac{\theta}{\theta_{0}}=\frac{T}{T_{0}}-\frac{\theta \phi}{v_{0}}
$$

or from $(55)$ that

$$
\frac{\theta}{\theta_{0}}=\frac{T}{T_{0}}-\frac{\theta \phi_{1}}{v_{0}}
$$

To use either of these equations for finding $\theta_{0}$, we set $\theta=\theta_{0}+$ roo and $T=T_{0}+$ roo. The value of $T_{0}$ is known; the value of $\theta_{0}$ to be used in finding the value of the correction term was taken to be $273^{\circ}$. I4 in a first approximation, and in a second approximation the value of $\theta_{0}$ found in the first approximation was used.

\section{VALUES FOUND FOR THE THERMODYNAMIC TEMPERATURE OF THE ICE POINT.}

The values found for $\theta_{0}$ by the several methods described in $\S 9$ are collected in Table VI.

For carbonic acid we find $\theta_{0}=273^{\circ}$. I 3 I from Chappuis' value of the coefficient of expansion at a pressure of $\mathrm{I} 377 \mathrm{~mm}$, and $\theta_{0}=273^{\circ}$. I I 2 from his value at a pressure of $998 \mathrm{~mm}$. The mean specific heat was taken from Lussana's data corrected to $50^{\circ}$ by Regnault's data. From Chappuis' value of the coefficient of expansion at a pressure of 5 I $8 \mathrm{~mm}$, the resulting value is $\theta_{0}=272.700$. The experimental results at this pressure were less concordant, and it has seemed justifiable to disregard this value in taking the mean. Since the value $\theta_{0}=273^{\circ}$. I 4 assumed in making the first approximation is so close to the resulting value, a second approximation is unnecessary. No computation was made by the second form of equation for $\mu$, since it does not fit the observations well for carbonic acid. 
In considering the values given in Table VI we may at the start confine our attention to the values obtained from the reduced equations for $\mu$, the other values having been computed and included in the table merely to illustrate the magnitude of the differences caused by the use of different methods of treating the same experimental data. A detailed examination of the experimental data does not

TABLE VI.

Temperature of the Ice Point.

\begin{tabular}{|c|c|c|c|c|}
\hline Method & Air & $\mathrm{N}_{2}$ & $\mathrm{H}_{2}$ & $\mathrm{CO}_{2}$ \\
\hline $\begin{array}{c}\qquad \begin{array}{c}\mu=a-b T \\
a \text { and } b \text { direct from observations.......... } \\
\mu=a-b T\end{array}\end{array}$ & 273.246 & & 273.056 & \\
\hline $\begin{array}{l}a \text { and } b \text { from equation (42) at } 20^{\circ} \text { and } 80^{\circ} \mathrm{C} . . \\
\qquad \mu=a / T^{2}\end{array}$ & 273.269 & 273.279 & 273.056 & \\
\hline $\begin{array}{l}a \text { from equation }(42) \ldots \ldots \ldots \ldots \ldots \ldots \\
\qquad \mu=\frac{a}{\theta-b}-c(\theta-b)-d\end{array}$ & 273.275 & 273.275 & & \\
\hline $\begin{array}{l}\text { fiirst approximation } . . . \ldots \ldots \ldots \\
\qquad \mu=\frac{a}{\theta-b}-c(\theta-b)-d\end{array}$ & 273.263 & 273.244 & 273.060 & 273.121 \\
\hline $\begin{array}{l}\text { second approximation } \ldots \ldots \\
\qquad \mu=\frac{a_{1}}{\theta-b_{1}}-d_{1} \\
\text { based on last value of } \theta_{0} \ldots \ldots\end{array}$ & 273.266 & 273.245 & 273.061 & \\
\hline
\end{tabular}

enable us to say whether the values for the different gases should be assigned equal weights or not. Using the means of the last two values for air, nitrogen, and hydrogen, the arithmetic mean is

$$
\theta_{0}=273^{\circ} \cdot 192
$$

while if the value for carbonic acid be included, the mean is

$$
\theta_{0}=273^{\circ} \cdot 174
$$


Another point of view is possible, however. If the discrepancies be assumed to be due, not to errors in the determination of the coefficients of expansion, but to errors in the elements which enter into the computation of the correction term which reduces $T_{0}$ to $\theta_{0}$, notably to errors in $\mu C_{p}$, it may be argued that the uncertainty of this correction is proportional to its total magnitude, and a weight may be assigned to each value proportional to the reciprocal of $\theta_{0}-T_{0}$. These differences $\theta_{0}-T_{0}$ are, for the four gases, approximately as $6: 6: \mathrm{I}: 42$. If weights be assigned in this way, the mean obtained is $\theta_{0}=273^{\circ}$ Io6, while if the weights are made proportional to the square roots of these reciprocals, the weighted mean is $\theta_{0}=273^{\circ}$. I 3 I. Since Chappuis' experiments show that hydrogen does not give so consistent results in the gas thermometer as nitrogen, it seems probable that these methods of weighting give too much importance to hydrogen.

All we can conclude from these values alone is that the value of $\theta_{0}$ is probably between $273^{\circ}$. I and $273^{\circ} .2$, but it is worth while to compare them with values obtained by other methods. The most interesting computation of this sort is that of D. Berthelot contained in Volume XIII of the Travaux et Mémoires of the International Bureau at Sèvres.

As was stated in $\S \mathrm{I}$, the problem of finding the relation of any gas scale to the thermodynamic scale-including the determination of $\theta_{0}$-is essentially the problem of investigating the departures of the gas from the ideal state and the computation of corrections for these departures. The usual method of doing this is to use the values of the Joule-Thomson effect. This is the method which has been pursued in the present paper, the only innovations introduced being in the treatment of the value of the Joule-Thomson effect where Kester's results on carbonic acid have become available, and in the use of Holborn and Austin's recently published results on the specific heat of nitrogen. But an entirely different mode of attack is possible. As was remarked in $\S 4$, the equations show that if the gas became infinitely rare, while $\mu C_{p}$ remained finite, the gas scale would become identical with the thermodynamic scale. Hence, if it be possible to find, by extrapolation from finite to infinitesimal pressures, the limiting values of the coefficients of expansion $a$, and 
of pressure $\beta$, these values should be identical with each other and with $\gamma$, the coefficient of expansion of the ideal gas, so that the thermodynamic scale would become known at once in its relation to the gas scales at ordinary pressures withont any consideration of either the Joule-Thomson effect or the specific heat. The general application of this method presupposes a knowledge of the equation of state of the gas for low pressures.

II. Berthelot has used this method with great success. For finding the value of $\theta_{0}$, the data needed are the values of $a$ and $\beta$ for any given pressure, and the compressibility at $0^{\circ}$. These quantities being known for nitrogen and hydrogen, from Chappuis' experiments, the assumption that the departures from Boyle's law remain uniform down to zero pressure makes it possible to compute immediately the value of $\gamma$, the mean coefficient of expansion of the ideal gas between the ice and steann points. The reciprocal of this is the desired value of $\theta_{0}$.

The mean of four independent and very consistent values obtained

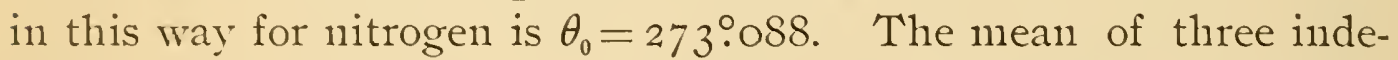
pendent and somewhat less consistent values for hydrogen is $\theta_{0}=$ $273^{\circ} .069$. The mean of all seven values is

$$
\theta_{0}=273: 079
$$

which II. Berthelot considers to be probably a little low but correct within 0 :O2.

II. Berthelot also computes the value of $\theta_{0}$, by the use of the Jonle-Thonnson effect, with the following results:

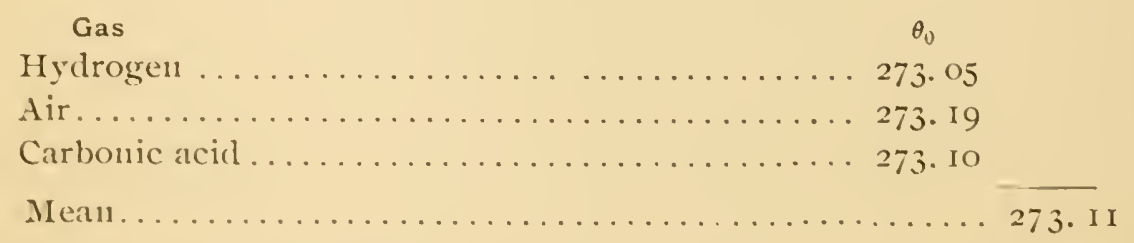

The values for hydrogen and carbonic acid are nearly the sane as those here obtained, namely, $273^{\circ} .05^{8}$ and $273^{\circ} . \mathrm{I} 2 \mathrm{r}$, while the value for air is sensibly lower than the value $273^{\circ} .27$ I obtained in the present work. The difference is probably clue to the different assumptions regarding the value of $\mu$. It has been pointed out $(\S \delta)$ 
that the equations used in the present work appear to represent the actual observations on the Jonle-Thonison effect somewhat better than M. Berthelot's equation. He gives no value for nitrogen, doubtless because at the time when his paper was published 110 direct measurements on the specific heat of nitrogen were available. The value for nitrogen based on the new data is nearly as high as that for air, namely, $273^{\circ} \cdot 25$, as compared with $273^{\circ} \cdot 27$. Assumining that the value for nitrogen, if computed by M. Berthelot's method, would also be 0.02 lower than that for the air, the mean for the four gases computed by his method would be $\theta_{0}=273^{\circ}$. 3 . There does not seem, on the whole, to be any sufficient reason for considering the valne for nitrogen to be less reliable than the others.

We may then collect the results as follows:

\begin{tabular}{|c|c|c|c|}
\hline Method & Value of $\theta_{0}$ & Gases & Author \\
\hline Extrapolation to $p=0$. & 273.079 & $\mathrm{H}_{2}$ and $\mathrm{N}_{2}$ & Berthelot \\
\hline Thermodynamic: $\mu=\frac{A}{\theta^{2}}-B$. . & 273.13 & $\mathrm{H}_{2}, \mathrm{~N}_{2}, \mathrm{CO}_{2}$, Air & " \\
\hline $\left.\begin{array}{c}\text { Thermodynamic: } \mu=\frac{a_{1}}{\theta-b_{1}}-d_{1} \\
\text { and } \mu=\frac{a}{\theta-b}-c(\theta-b)-d\end{array}\right\}$ & 273.174 & $\mathrm{H}_{2}, \mathrm{~N}_{2}, \mathrm{CO}_{2}$, Air & Buckingham \\
\hline
\end{tabular}

The mean of these,

$$
\theta_{0}=273^{\circ} \cdot \mathrm{I} 3
$$

is probably not far from the true value. It remains for future experiments on the value of $\mu C_{p}$ to decide whether the consistently high values obtained by the thermodynamic method of computation for air and nitrogen are erroneous, or whether there is some as yet undetected error in the experimental data from which M. Berthelot has obtained his beautifully consistent but much lower results for hydrogen and nitrogen.

$$
23835-07-8
$$


11. CORRECTIONS OF THE CONSTANT-PRESSURE HYDROGEN AND NITROGEN THERMOMETERS BETWEEN THE ICE AND STEAM POINTS.

By the "correction of the gas scale to the thermodynamic scale" is meant, not the difference between the absolute temperatures by the two scales, a quantity which would be uncertain by the whole amount of the uncertainty in $\theta_{0}$, but the difference between the centigrade scale of the gas thermometer and the centigrade thermodynamic scale as defined in $\S \mathrm{I}$. These corrections are small between $0^{\circ}$ and $100^{\circ}$ and they may be found with an accuracy exceeding that attainable in the use of the gas thermometer by the simplest of the methods described in $\S 9$. From the assumption that the Jonle-Thomson effect might be treated as a linear function of the temperature, or that

$$
\mu=a-b T
$$

we obtained the equation

$$
\frac{\theta}{\theta_{0}}=\left[\frac{T+A^{\prime} B}{T_{0}+A^{\prime} B}\right]^{v_{0} / B}
$$

where

$$
\left.\begin{array}{l}
A=a T_{0} C_{p} \\
B=v_{0}-b T_{0} C_{p}
\end{array}\right\}
$$

If the values of $a$ and $b$ be found from the general equation (40) by making the straight line intersect the curve at $20^{\circ}$ and $80^{\circ}$, the corrections may easily be computed.

The resulting values for the constant-pressure hydrogen thermometer are given in Table VII, in comparison with values computed by other writers. Column I gives the temperature; column 2, the corrections computed by Rose-Innes ${ }^{20}$; columns 3 and 4, the corrections computed by Callendar ${ }^{21}$ by two methods; column 5 , the values according to D. Berthelot 2 ; column 6 , the values computed by the method just described; colunn 7 , the values given by the same nuethod when $a$ and $b$ are found directly from the equations given in $\S 6$. The values computed by Rose-Innes and Callendar are given in their

${ }^{20}$ Phil. Mag. (6), 2, p). г30; r 90 I.

${ }^{21}$ Phil. Mag. (6), 5, p. 4S; 1903.

${ }^{22}$ 'Trav. et MIem. Bur. Int. XIII, 1903. 
papers for a pressure of $760 \mathrm{~mm}$; they have therefore been multiplied by $\frac{4}{3}$, so that as given in Table VII all the corrections refer to a pressure of $1000 \mathrm{~mm}$.

The figures in columns $2,3,6$, and 7 , obtained from the consideration of the Joule-Thomson effect, are all sensibly in agreement. The values in columns 4 and 5 , obtained without using the Joule-Thom-

\section{TABLE VII.}

Corrections of the Constant-Pressure Hydrogen Scale, $\pi=1000 \mathrm{~mm}$.

[The corrections are all to be subtracted.]

\begin{tabular}{|c|c|c|c|c|c|c|}
\hline I & 2 & 3 & 4 & 5 & 6 & 7 \\
\hline$t^{\circ} \mathrm{C}$ & $\begin{array}{l}\text { Rose-Innes } \\
\text { IgoI }\end{array}$ & $\begin{array}{c}\text { Callendar, I } \\
\text { rgo3 }\end{array}$ & $\begin{array}{l}\text { Callendar, II } \\
\text { Igo3 }\end{array}$ & $\begin{array}{l}\text { D. Berthelot } \\
\text { Igo3 }\end{array}$ & E. B., I & E. B.,II \\
\hline 10 & 0.0015 & 0.0015 & 0.0007 & $\ldots \ldots$ & 0.0015 & 0.0013 \\
\hline 20 & 0.0024 & 0.0025 & 0.0013 & 0.0008 & 0.0026 & 0.0023 \\
\hline 30 & 0.0031 & 0.0032 & 0.0016 & 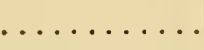 & 0.0033 & 0.0029 \\
\hline 40 & 0.0033 & 0.0035 & 0.0018 & 0.0010 & 0.0037 & 0.0032 \\
\hline 50 & 0.0033 & 0.0035 & 0.0018 & $\ldots \ldots$ & 0.0039 & 0.0034 \\
\hline 60 & 0.0031 & 0.0033 & 0.0016 & 0.0009 & 0.0037 & 0.0032 \\
\hline 70 & 0.0025 & 0.0027 & 0.0014 & & 0.0031 & 0.0028 \\
\hline 80 & 0.0019 & 0.0020 & 0.0010 & 0.0005 & 0.0024 & 0.0022 \\
\hline 90 & 0.0011 & 0.0011 & 0.0005 & & 0.0013 & 0.0013 \\
\hline
\end{tabular}

son effect, by general considerations on the equation of state, are, respectively, about $1 / 2$ and $1 / 4$ of the mean of the others. The absolute values are thus in doubt, but as the largest of all the values is less than $0: 004$ the corrections may be considered negligible.

In Table VIII are given similar values for the constant-pressure nitrogen scale. As before, all the corrections are given for a pressure of $1000 \mathrm{~mm}$. The figures in column 3 are for air, but the corrections computed by the same method for nitrogen would doubtless be very nearly the same.

The agreement among these sets of value is relatively much closer than was the case for hydrogen, though since the values are larger, the absolute magnitude of the divergence is also a trifle larger, the greatest difference being about $0: 008$. The mean of columns 2, 
4, 5, and 6, given in column 7 , is everywhere within 0 :oor of Berthelot's value and may be regarded as correct within the present limits of experimental precision in gas themometry.

\section{TABLE VIII.}

Corrections of the Constant-Pressure Nitrogen Scale, $\pi=$ Iooo mm.

[The corrections are all to be subtracted.]

\begin{tabular}{|c|c|c|c|c|c|c|}
\hline$I$ & 2 & 3 & 4 & 5 & 6 & 7 \\
\hline$t^{\prime \prime} \mathrm{C}$ & $\begin{array}{c}\text { Rose-Innes } \\
\text { rgor }\end{array}$ & $\begin{array}{c}\text { Callendar, I } \\
\text { rgo3 } \\
\text { (Air) }\end{array}$ & $\begin{array}{c}\text { Callendar, II } \\
\text { I903 }\end{array}$ & $\begin{array}{c}\text { D. Berthelot } \\
1903\end{array}$ & E. B. & $\begin{array}{l}\text { Mean } \\
\text { (omitting } \\
\text { col. 3) }\end{array}$ \\
\hline 10 & 0.0120 & 0.0097 & 0.0109 & 0.010 & 0.0078 & 0.010 \\
\hline 20 & 0.0205 & 0.0163 & 0.0188 & 0.017 & 0.0137 & 0.017 \\
\hline 30 & 0.0261 & 0.0207 & 0.0236 & 0.022 & 0.0179 & 0.022 \\
\hline 40 & 0.0288 & 0.0225 & 0.0260 & 0.024 & 0.0203 & 0.025 \\
\hline 50 & 0.0289 & 0.0226 & 0.0260 & 0.024 & 0.0209 & 0.025 \\
\hline 60 & 0.0269 & 0.0207 & 0.0240 & 0.022 & 0.0198 & 0.023 \\
\hline 70 & 0.0228 & 0.0176 & 0.0204 & 0.019 & 0.0172 & 0.020 \\
\hline 80 & 0.0168 & 0.0131 & 0.0151 & 0.014 & 0.0129 & 0.015 \\
\hline 90 & 0.0092 & 0.0069 & 0.0081 & 0.007 & 0.0071 & 0.008 \\
\hline
\end{tabular}

12. COMPUTATION OF THE CORRECTIONS FOR TEMPERATURES OUTSIDE THE FUNDAMENTAL INTERVAL.

Since the methods of computing used in this paper involve the nse of the Jonle-Thonison effect, and since, with the exception of Olszewski's value for the inversion point of hydrogen, the data on this quantity have been obtained from experinents at temperatures between $\mathrm{O}^{\circ} \mathrm{C}$ and $100^{\circ} \mathrm{C}$, any computation of the relations of the gas scales to the thermodynamic scale at temperatures ontside this interval must involve an extrapolation. The general reduced equation (40) or (4I) affords the means of making this extrapolation with a considerable degree of probability. It embraces observations at temperatures all the way from a little below the critical temperature to somewhat over eleven times the critical temperature. For the purpose of extrapolation it will now be assunned that the corresponding nureduced equation for each gas is valid, not nnerely between $\mathrm{O}^{\circ}$ and $100^{\circ}$, but from the critical temperature of that gas to at 
least eleven times the critical temperature. On this basis corrections will be computed for the hydrogen thermometer down to $-240^{\circ} \mathrm{C}$,

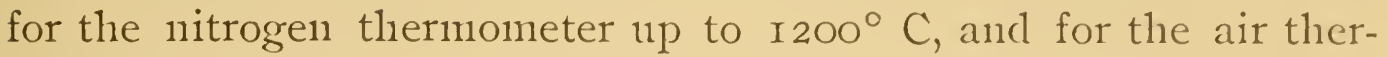
mometer at $445^{\circ}$ In these computations equations (54) and (57) will be used, and these must now be considered more closely.

In the equation

$$
\mu=\frac{a}{\tau-b}-c(\tau-b)-d
$$

the temperature $\tau$ is not the temperature by either the constantvolume or the constant-pressure scale of the gas in question, but the centigrade temperature, as stated by various observers, plus $273^{\circ}$. It may safely be assumed that the tabulated values for the temperatures of the observations on $\mu$, as well as for the critical temperatures, are given in terms of the normal constant-volume hydrogen scale, and that this scale agrees with the centigrade thermodynamic scale, both within the errors of the observations. In the computation by means of equations (54) and (57) this temperature $\tau$ is identified with the absolute thermodynamic temperature $\theta$. But it now appears that the absolute thermodynamic temperature of the ice point is somewhat above $273^{\circ}$, and the value $273^{\circ} .13$ appears most probable. Strictly speaking, therefore, a second approximation ought now to be made. Instead of adding $273^{\circ}$ to the observed values in getting the reduced temperatures and cooling effects, the computation should be revised, using $273^{\circ}$. 3 instead of $273^{\circ}$. The coefficients of the reduced equation $\mu^{\prime}=f\left(\tau^{\prime}\right)$ ought to be redetermined, and from these, the values of the coefficients in the unreduced equation for each gas. But it is quite certain that the accuracy of the experimental data does not warrant this and that the improvement in accuracy would be illusory.

All the corrections between $0^{\circ}$ and $100^{\circ}$ are so small that it is of little importance what method is used for computing them, but when it is a question of wide extrapolation, more caution is needed. The one essential condition is that the corrections shall vanish at $0^{\circ}$ and $100^{\circ}$. Now, when equations (54) and (57) were used to find the value of the ice point for nitrogen they gave $\theta_{0}=273.244$. It is evident therefore that if the same values of $a, b, c, d$ be adopted, but $\theta_{0}$ be arbitrarily set equal to 273.13 or any other value than 273.244 , the 
correction will not vanish at $100^{\circ}$. Hence the values of $a, b, c$, and $d$ already given for the three gases do not, when taken in conjunction with the single value $\theta_{0}=273$. I 3 , satisfy the essential condition of making the corrections vanish at $100^{\circ}$.

Two methods of procedure suggest themselves. We may take the values of the constants already determined and use them in connection with the value of $\theta_{0}$ found for each particular gas from the condition that the correction shall vanish at $100^{\circ}$ as well as at $0^{\circ}$. Or we inight adopt the value $\theta_{0}=273$. I 3 for all the gases and adjust the constants for each gas so as to fulfill the condition of giving no correction at $100^{\circ}$. In view of the fact that the values of $\mu$ and therefore of the constants $a, b, c, d$ are somewhat in doubt, and that there certainly is one definite true value of $\theta_{0}$, even if it be not precisely $273^{\circ}$ I 3 , the second method seems more logical. But since there is no way of telling just what the errors are, and therefore no satisfactory way of adjusting the constants, the safest method is to keep strictly to the results of experiment and use for each gas the separate value of $\theta_{0}$ found for it. This method has therefore been pursued.

In Table IX are given the values of the coefficients of equation (54) which were used in computing the temperature of the ice point as described in $\S 9$. They were obtained from the values of $a, b, c, d$ (see Table V) found for each gas from the reduced equation (40) as described in $\S 7$. The corresponding values of $\theta_{0}$ are also given, so that the figures for each gas form a consistent system of values which may be used in the first of the two methods of extrapolation just considered.

TABLE IX.

Values of the Constants of Equation (54).

\begin{tabular}{c|c|c|c}
\hline $\log _{10}\left(\frac{a}{b}-c b+d\right)$ & Air & Nitrogen & Hydrogen \\
\hline $\log _{10} \frac{a}{b^{2}}$ & $\overline{6} .13710$ & $\overline{6} .18852$ & $\overline{7} .82499$ \\
$\log _{10^{6}}$ & $\overline{8} .37367$ & $\overline{8} .44172$ & $\overline{8} .68026$ \\
$\theta_{0}$ & $\overline{11.77897}$ & $\overline{11.84702}$ & $\overline{10.08556}$ \\
& 273.266 & 273.245 & 273.061 \\
\hline
\end{tabular}




\section{CONSTANT-PRESSURE CORRECTIONS FOR HYDROGEN AND NITROGEN OUTSIDE THE FUNDAMENTAL INTERVAL.}

The computation of the corrections between $0^{\circ}$ and $100^{\circ}$ may be made by very simple methods. The values tabulated in $\S$ II are all small and the results from different methods agree closely. Equations (54) and (57), together with the constants given in Table IX, have also been used to compute the corrections of the constantpressure hydrogen and nitrogen thermometers for several points. The same corrections have been computed by Callendar by two methods, and by Berthelot. The four sets of values for hydrogen are collected in Table $\mathrm{X}$, Callendar's values for the pressure $\pi=760 \mathrm{~mm}$ having been multiplied by $\frac{4}{3}$ to make them comparable with the others. The two values given in the column headed "E. B., II" were obtained by equations $(55)$ and (58), which are based on the value $p_{c}=\mathrm{I} 3$ atmospheres for hydrogen, and were computed for the sake of finding how much they differed from the values given by equations (54) and (57) based on $p_{c}=19.4$ atmospheres for hydrogen.

TABLE $X$.

Constant-Pressure Corrections for Hydrogen, $\pi=1000 \mathrm{~mm}$.

\begin{tabular}{|c|c|c|c|c|c|}
\hline$t^{\circ} \mathrm{C}$ & Callendar, I & Callendar, II & D. Berthelot & E. B., I & E. B., II \\
\hline-240 & & 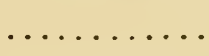 & +1.368 & +0.833 & \\
\hline-200 & +0.781 & +0.268 & +0.250 & +0.271 & +0.349 \\
\hline-150 & +0.211 & +0.085 & +0.060 & +0.111 & \\
\hline-100 & +0.066 & +0.029 & +0.021 & +0.046 & \\
\hline+50 & -0.0035 & -0.0018 & -0.0011 & -0.0050 & \\
\hline+200 & +0.017 & +0.009 & +0.005 & +0.024 & \\
\hline+300 & +0.041 & +0.022 & $\ldots \ldots$ & +0.064 & \\
\hline+400 & $\ldots \ldots$ & $\ldots \ldots \ldots$ & +0.021 & +0.110 & +0.068 \\
\hline+450 & +0.081 & +0.045 & & & \\
\hline
\end{tabular}

At first sight there appears to be hardly any agreement between the various values in this table except in the respect that most if not all of the corrections are within the experimental errors unavoidable in gas thermometry at the present time. A closer inspection shows that there is a fair agreement, for the low temperatures, 
between the columns headed "Callendar, II, "D. Berthelot," and "E. B., I," and the mean of these sets of values is probably the best indication we have at present of the true values. At the higher temperatures the divergence is much greater. As regards the values computed by equations (54) and (57), it may be said that they are based on an extrapolation in every sense, since with hydrogen, which has the lowest critical temperature of all the gases considered, there is no possibility of using the law of corresponding states for checking the values at temperatures above $\mathrm{IOO}^{\circ}$ by comparison with other gases. Somewhat similar remarks are applicable to Berthelot's results, although since his computations are based on an apparently very satisfactory form of the equation of state and not on a confessedly empirical equation for $\mu$, they appear to deserve more confidence than the values computed by the present writer. The curves plotted from the various sets of figures have a general similarity of form but do not lie close together. It is obvious that there are inconsistencies in the experimental data on the behavior of hydrogen, which it remains for future experiment, particularly on the Joule-Thomson effect and on the possible influence of adsorption on the coefficients of expansion and pressure, to clear up.

The same method of computation has been pursued for nitrogen, and the results, reduced by $\mathrm{r} / 500$ so as to make them applicable to a pressure of IOOO mm., together with those of Callendar and Berthelot, are collected in Table XI.

TABLE XI.

Constant-Pressure Corrections for Nitrogen, $\pi=1000 \mathrm{~mm}$.

\begin{tabular}{|c|c|c|c|c|}
\hline$t^{\circ} \mathrm{C}$ & Callendar, II & D. Berthelot & E. B., I & E. B., II \\
\hline-100 & +0.437 & +0.469 & +0.326 & +0.348 \\
\hline+50 & -0.025 & -0.024 & -0.0190 & \\
\hline 200 & +0.135 & +0.113 & +0.105 & \\
\hline 400 & $\ldots$ & +0.457 & +0.433 & -0.456 \\
\hline 450 & +0.660 & . & $\ldots$ & $\cdots$ \\
\hline 600 & & +0.844 & +0.827 & \\
\hline 800 & $\ldots \ldots$ & +1.248 & +1.262 & …… \\
\hline 1000 & +2.047 & +1.654 & +1.706 & +1.721 \\
\hline 1200 & & $\ldots$ & +2.157 & -+2.170 \\
\hline
\end{tabular}


$\stackrel{0}{0}$

$\stackrel{\circ}{\circ}$

๙ั

¿

:

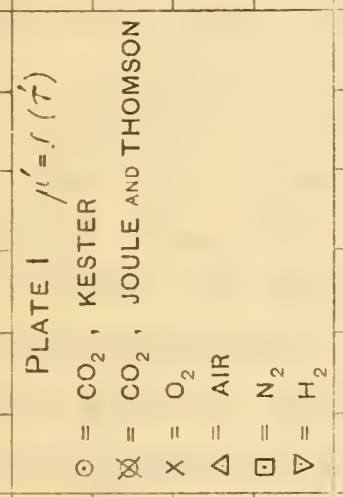

$=$

$\stackrel{-}{\circ}$

$\frac{1}{9}$

10



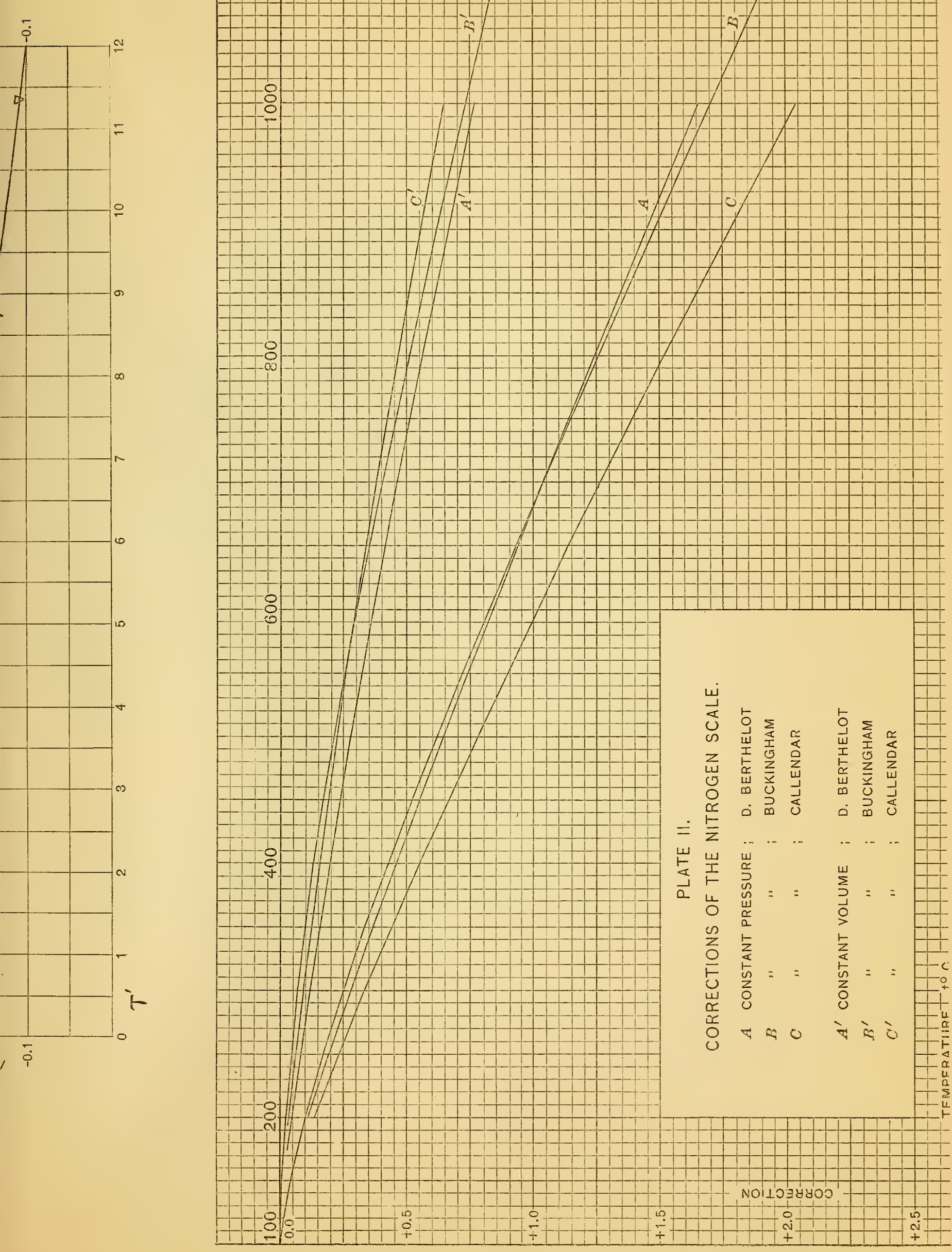




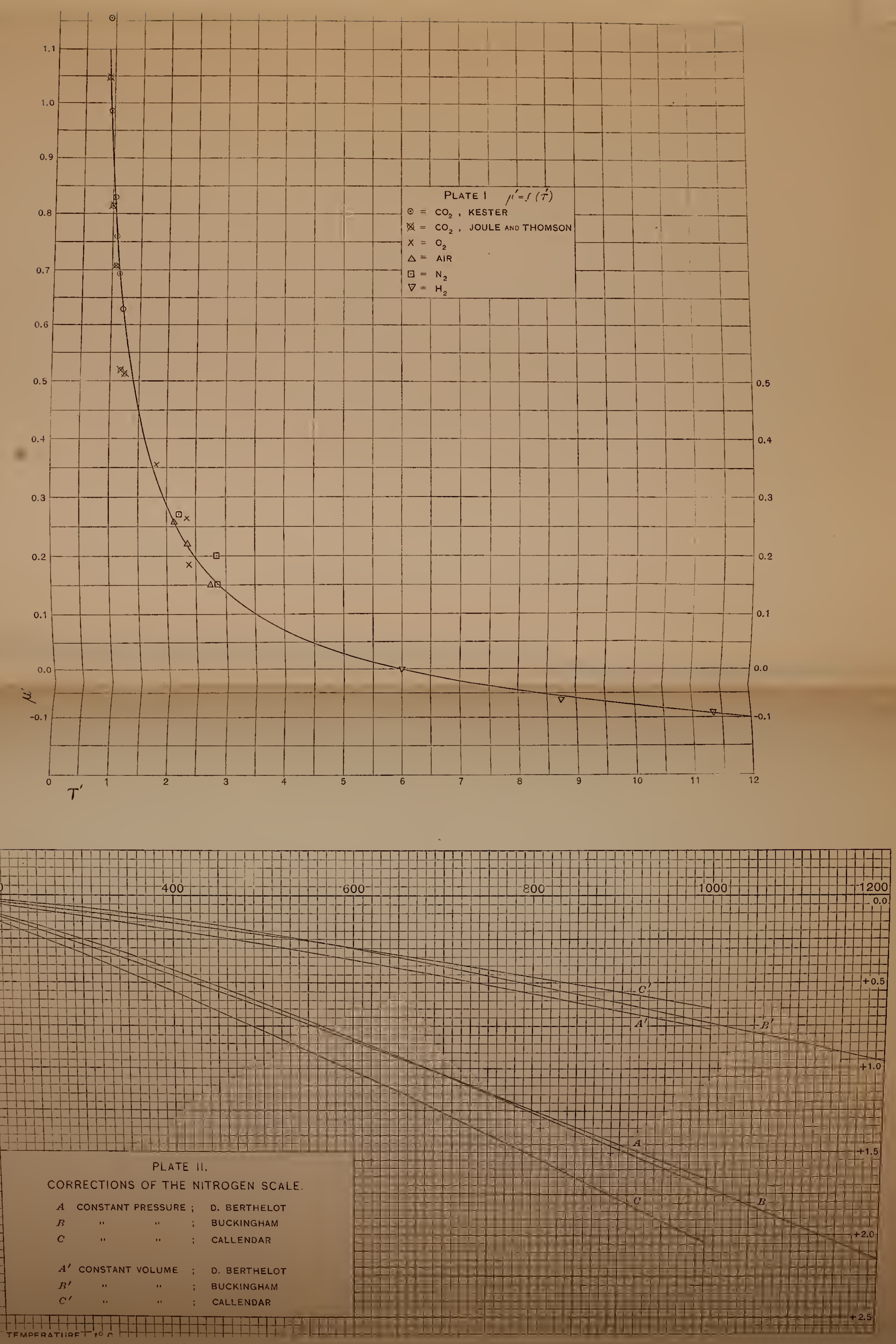


Since the temperatures considered in this table all lie within the limits of the curve on Plate I, i. e., with the exception of $\mathrm{I}_{200^{\circ}}$, within the limits where the law of corresponding states affords us the support of comparison between different gases, the results would appear a priori to deserve more confidence than those for hydrogen. We find accordingly that the agreement is satisfactory, especially between Berthelot's values and those of the present paper. The two methods here pursued give nearly the same results, although the equations, namely (4O) and (4I), are based on two quite different values of the critical pressure of hydrogen, which probably include the true value between them. The greatest discrepancy between M. Berthelot's results and my own is at the lowest temperature. This was to have been expected. For the equation of state, upon the consideration of which M. Berthelot's computations are founded, leads to a value for $\mu$ which differs from the ones here used more and more as the temperature is lower and lower. Fortunately, this divergence of opinion as to the better form of equation for $\mu$ is of little importance, because there is little likelihood that the nitrogen thermometer will be used for low temperatures. Callendar's results are somewhat larger, but the difference of 0.4 at $1000^{\circ}$ is small compared with the experimental errors at this temperature. On Plate II the three sets of results are plotted. Curve (A) represents Berthelot's values; curve (B), those of the present paper computed by the first method; and curve (C), Callendar's values. It seems probable that the mean of the three gives the true values with a precision not likely to be soon surpassed in experimental work.

\section{THE BOILING POINT OF SULPHUR.}

If the values given in Table IX be used in equations (54) and (57) to find the correction of the constant-pressure air thermometer at $t_{\theta}=445^{\circ}$, the result is $t_{p}=444^{\circ} \cdot 5 \circ 3$, hence the correction of the constant-pressure air thermometer for which $\pi=$ IOOI $\mathrm{mm}$ is, at this temperature,

$$
v_{\theta}-t_{p}=+0.497
$$

The value of $\left[C_{p}\right]_{0}^{\mathrm{t}}$ used in the computation was found by taking Lussana's value for air at a pressure of Ioor mm and assuming that the specific heat of air varies with the temperature in the same way 
as Holborn and Austin found for nitrogen. Increasing the specific heat by I per cent increases the correction by o:or. An independent computation, based on $\theta_{0}=273^{\circ} \cdot 26 \mathrm{I} 3$, gave $+0: 504$ for the correction, or sensibly the same.

Callendar and Griffiths ${ }^{23}$, using a constant-pressure air thermometer at atmospheric pressure, found for the boiling point of sulphur the value $444^{\circ} \cdot 53$. At this pressure the correction as just given becomes $3 / 4 \times 0.497=0.37$; hence, according to this determination, the boiling point of sulphur on the centigrade thermodynamic scale is

$$
\text { (S. B. P. })_{\theta}=444^{\circ} \cdot 90
$$

Chappuis and Harker ${ }^{24}$, using a constant-volume nitrogen thermometer with an initial pressure of $528 \mathrm{~mm}$, found for the same point the value $445^{\circ} \cdot 27$. Somewhat later, Chappuis ${ }^{25}$, after the publication of Holborn and Day's results on the expansion of porcelain, concludes that this value should be lowered by about $0: 5$, which would bring it to $444^{\circ} 77$. In $\S_{17}$ it will be shown that the correction for this thermometer is +0.09 , which gives for the value of the boiling point of sulphur on the centigrade thermodynamic scale, according to the determination of Chappuis and Harker,

$$
\text { (S. B. P. })_{\theta}=444: 86
$$

The agreement of these two values, which is much better than could have been anticipated, in view of the experimental difficulties, is the best test at present possible of our methods of computing the corrections in question.

\section{THE RELATION OF THE CONSTANT-VOLUME AND CONSTANT-PRESSURE SCALES.}

It has already been pointed out $\left(\S_{3}\right)$ that to treat the theory of the constant-volume thermometer by means of the Joule-Thomson effect we must have a complete knowledge of the compressibility of the gas throughout the range of temperature under consideration, although since the terms involving the compressibility are small, a merely approximate knowledge is sufficient. But if we have this

\footnotetext{
${ }^{23}$ Phil. Trans. I89i, A, p. II 9.

${ }^{24}$ Phil. Trans. I900, A, p. Io2.

${ }^{25}$ Phil. Mag. (6), 3, p. 246; 1902.
} 
knowledge of the compressibility, it is possible to find the relation of the constant-volume and constant-pressure scales; and since we have already, by a plansible method, found the thermodynamic corrections of the constant-pressure scale, we may deduce the corrections of the constant-volume scale from them by a process which is much simpler than integrating the general equations of the constantvolume thermometer given in $\S 3$. In either case, some hypothesis as to the equation of state must be made, and the correctness of the results obtained depends on how near the particular hypothesis comes to the truth. Since the corrections of the constant-volume thermometer have been computed by other writers by other methods, it seems worth while to use the simple method just suggested and compare the results with theirs. This will now be done.

If $t_{v}$ be the centigrade temperature by the constant-volume gas scale, the gas being at the constant specific volume $v=\phi$ and the pressure at the ice point being $p_{0}=\pi$, we have, by definition,

$$
p=\pi\left(\mathrm{I}+\beta t_{v}\right)
$$

If $t_{p}$ be the centigrade temperature by the constant-pressure gas scale, the gas being under the constant pressure $p=\pi$ and the specific volume at the ice point being $v_{0}=\phi$, we have, by definition,

$$
v=\phi\left(\mathrm{I}+a t_{p}\right)
$$

Let the departures of the gas from Boyle's law be represented as usual by the equation

$$
p_{2} v_{2}=p_{1} v_{1}\left[\mathrm{I}+K\left(p_{2}-p_{1}\right)\right]
$$

in which $K$ is the average value, between $p_{2}$ and $p_{1}$, of $\frac{I}{p v} \frac{\partial}{\partial p}(p v)$ and is some function of the temperature. Let the gas be at the temperature represented on the two scales by $t_{v}$ and $t_{p}$, respectively. Then, if we let $p_{2}=p$ and $v_{1}=\phi$, we have, by substituting in equation (65) from equations (63) and (64),

$$
\pi\left(\mathrm{I}+\beta t_{v}\right) \phi=\pi \phi\left(\mathrm{I}+a t_{p}\right)\left(\mathrm{I}+K \pi \beta t_{v}\right)
$$

whence

$$
t_{p}=t_{v} \frac{\beta}{a} \frac{\mathrm{I}-K \pi}{\mathrm{I}+K \pi \beta t_{v}}
$$


If, therefore, we have the values of the coefficient of expansion $a$, the coefficient of pressure $\beta$, and $K$ as a function of $t_{v}$, we may at once find the value $t_{p}$ on the constant-pressure scale, of any temperature which on the constant-volume scale is denoted by $t_{v}$. Equation (66) is entirely rigorous, being a direct consequence of the definitions of $t_{v}, t_{m}$, and $K$ contained in equations (63), (64), and (65). To make use of it we have to make some hypothesis about the variation of $K$ with $t_{v}$, since experiments on compressibility at the pressures involved in gas thermometry have been made at only a very few temperatures.

16. ASSUMPTIONS REGARDING THE VARIATION OF $K$ WITH TEMPERATURE.

If in equation (65) we let $p_{1}=p$ and $p_{2}=p+\delta p$ we have

$$
p v+\delta(p v)=p v+K p v \delta p
$$

whence

$$
K=\frac{\mathrm{I}}{p v} \frac{\partial}{\partial p}(b v)
$$

We also liave

$$
\frac{\partial}{\partial p}(p v)=v^{\prime}+p \frac{\partial v}{\partial p}
$$

The first step is to assume some form of equation of state as valid throughout the small range of pressures under consideration.

Van der Waals's equation,

$$
p=\frac{R \theta}{v-b}-\frac{a}{v^{2}}
$$

wonld indicate that the centigrade constant-volnme scale was identical with the centigrade thermodynamic scale. Since the constantvolume scales of different gases do not agree precisely, as shown by Chappuis' experinents, van der Waals's equation must be considered inadequate.

'Tlie first equation of Clausins, nanely,

$$
\left[p+\frac{a}{\theta(v+c)^{2}}\right](v-b)=R \theta
$$


may be made to represent the properties of gases more closely than that of van der Waals. From this we obtain

$$
\frac{\partial}{\partial p}(p v)=\frac{a(v-c)(v-b)^{2}-R \theta^{2}(v+c)^{3} b}{2 a(v-b)^{2}-R \theta^{2}(v+c)^{3}}
$$

If, while $\theta$ remains finite, the pressure approaches zero, so that $v$ becomes infinite, equation ( $7 \mathrm{I})$ approaches the form

$$
\frac{\partial}{\partial p}(p v)=b-\frac{a}{R} \frac{\mathrm{I}}{\theta^{2}}
$$

We next assume that the value of $\frac{\partial}{\partial p}(p v)$ is sensibly constant for pressures between zero and the highest pressure to be considered, which is only about 5.5 meters of mercury. This amounts to assuming that within this small range of pressures the isothermal curves $p v=f(\not p)$ are sensibly straight lines. Experiment shows them to be very nearly straight for the more nearly ideal gases, such as hydrogen and nitrogen, so far as the experiments have gone, so that this assumption is a legitimate expression of an experimental approximation. We may also safely assume that whatever be the real form of the equation of state, the gas approaches the ideal state as a limit when the pressure approaches zero. Since the absolute thermodynamic scale is identical with the scale of an ideal gas, we may write, for $p=0$,

$$
\operatorname{limit} \frac{(p v)}{(p v)_{0}}=\frac{\theta}{\theta_{0}}
$$

and if we then take as our unit of $p v$ the limiting value of $p v$ at the ice point $\theta_{0}$, we have for any other temperature $\theta$,

$$
\operatorname{limit}(p v)=\frac{\theta}{\theta_{0}}
$$

Since $K$ is itself a very small quantity, $p v$ does not vary much along each isothermal within the given range of pressures, and we may use the value given by equation (73) in equation (67). We then have finally, for the value of $K$ obtained from equations $(67)$ and (70),

$$
K_{\theta}=\frac{\theta_{0}}{\theta}\left(b-\frac{a}{R} \frac{\mathrm{I}}{\theta^{2}}\right)
$$


If we have experimental data on the values of $K$ for two different temperatures, of whicl the values $\theta_{0}$ and $\theta$ are known approximately, the constants $\frac{a}{R}$ and $b$ may be determined and an equation set $11 \mathrm{p}$ for computing the value of the coefficient $K$ at all other temperatures.

A large part of the paper of Berthelot ${ }^{26}$, which has so frequently been referred to, is devoted to the establishment and verification of an equation of state. This equation is very similar to that of Clansins and leads to the same result, namely, equation (74), for the limiting value of the coefficient $K$. M. Berthelot's paper was not received until after the foregoing considerations had been developed, and it was gratifying to find that the equation employed for computation was one that had already been used successfully.

According to the experiments of Chappuis on nitrogen we have, per meter of mercury,

$$
\left[\frac{\partial}{\partial p}(p v)\right]_{0}=-0.00057 \text { I } ;\left[\frac{\partial}{\partial p}(p v)\right]_{100}=+0.000347
$$

the value of $(p v)$ at $o^{\circ}$ and I meter of mercury being taken equal to unity. Since it is convenient in using equation (66) to have $\pi=\mathrm{I}$, and since all the other quantities involved refer, for nitrogen, to a pressure of $1002 \mathrm{~mm}$, we have merely to increase the above values by $\mathrm{I} / 500$. If we also set $\theta_{0}=273^{\circ} \cdot 2$, we have

$$
\begin{array}{ll}
\theta_{0}=273.2 & K_{0}=-0.0005723 \\
\theta_{100}=373.2 & K_{100}=+0.0002545
\end{array}
$$

If these figures be substituted in equation (74), they give

$$
\begin{aligned}
\frac{a}{R} & =147.95 \\
b & =0.001410 .
\end{aligned}
$$

If these values be used in the well-known relations ${ }^{2 \pi}$ deduced from equation (70), to compute the critical pressure from the critical temperature, they give

${ }^{26}$ 'Trav. et Mém. Bur. Int. XIII; I 903.

${ }^{27}$ See I'reston's Theory of Heat, 2 d ed., p. 5 I2. 


$$
\begin{array}{ccc} 
& \text { Calculated } & \text { Observed } \\
p_{c} \text { (atmospheres) } & 28.9 & 33.6
\end{array}
$$

In view of the small range of pressures used, the smallness of the values of $K$, and the extent of the extrapolation, the agreement of the observed and computed values is remarkably good and bears witness to the admirable precision of Chappuis' work.

We have, then, to make use of the equation

$$
K_{t}=\frac{273.2}{273.2+t}\left[0.001410-\frac{147.95}{(273.2+t)^{2}}\right]
$$

\section{THE CORRECTIONS OF THE CONSTANT-VOLUME NITROGEN THER- MOMETER.}

Having obtained an equation for $K$, it is now an easy matter by means of equation (66) to find the relation of $t_{p}$ and $t_{v}$. Since the equation for $K$ is given in terms of $1002 \mathrm{~mm}$ as the unit of pressure. equation (66) takes the form

$$
t_{p}=t_{v} \frac{\beta}{a} \frac{\mathrm{I}-K}{\mathrm{I}+K \beta t_{v}}
$$

Chappuis' values of $a$ and $\beta$ are

whence we have

$$
\begin{aligned}
& \beta=0.00367442 \\
& a=0.003673 \mathrm{I} 5
\end{aligned}
$$

$$
\frac{\beta}{a}=\mathrm{I} .000346
$$

and the equation to be used in computation is

$$
t_{p}=\mathrm{I} .000346 \frac{\mathrm{I}-K}{\mathrm{I}+K \beta t_{v}} t_{v}
$$

where $K$ is to be found from equation (75).

The results are shown in Table XII. In column I are given the values of $t_{v}$, the temperature by the centigrade constant-volume scale; in column 2, the values of $K$ computed by equation (75); in column 3 , the corresponding values of $t_{p}$ computed by equation $(76)$; in column 4 , the difference $t_{v}-t_{p}$; in column 5 , the corrections, for a pressure $\pi=1002 \mathrm{~mm}$, to be applied to the centigrade constant- 
pressure scale; in column 6, the resulting correction to be applied to the centigrade constant-rolume scale to reduce it to the centigrade thermodynamic scale. The value $t_{\theta}-t_{v}=-0.059$ at $-100^{\circ}$ is undoubtedly erroneous. This may be due to the failure of equation (75) to represent the compressibility at low temperatures, or it may be that the constant-pressure correction at this temperature as computed is too small. If the mean of Berthelot's and Callendar's corrections for the constant-pressure scale at this point be used, namely, $t_{\theta}-t_{p}+0.453$ instead of +0.326 , we have $t_{\theta}-t_{v}=+0.068$

\section{TABLE XII.}

Comparison of Constant-Volume and Constant-Pressure Scales for Nitrogen, $\pi=p_{0}=1002 \mathrm{~mm}$.

\begin{tabular}{|c|c|c|c|c|c|}
\hline I & 2 & 3 & 4 & 5 & 6 \\
\hline$t_{v}^{\circ}{ }_{v}{ }^{\circ}$ & $K^{\prime}$ & $t_{p}$ & $t_{v}-t_{p}$ & $t_{\theta}-t_{p}$ & $t_{\theta}-t_{v}$ \\
\hline-100 & -0.005555 & -100.385 & +0.385 & +0.326 & -0.059 \\
\hline+50 & -0.000005 & +50.0176 & -0.0176 & -0.0190 & -0.0014 \\
\hline 200 & +0.000433 & 199.919 & +0.081 & +0.105 & +0.024 \\
\hline 400 & +0.000440 & 399.704 & +0.296 & +0.435 & +0.139 \\
\hline 600 & +0.000380 & 599.476 & +0.524 & +0.830 & +0.306 \\
\hline 800 & +0.000326 & 799.250 & +0.750 & +1.265 & +0.515 \\
\hline 1000 & +0.000283 & 999.024 & +0.976 & +1.711 & +0.735 \\
\hline 1200 & +0.000249 & 1198.800 & +1.200 & +2.163 & +0.963 \\
\hline
\end{tabular}

In Table XIII is given a comparison of different values for the constant-volume nitrogen corrections. In colunnm I are the centigrade temperatures; in column 2, the corrections as computed by Callendar; in column 3, the corrections according to Berthelot; in column 4, the values from Table XII reduced by I/500 so as to make them applicable for an initial pressure $p_{0}=$ I 00011111 .

The interesting part of the table is that relating to the ligher temperatures. The values from $200^{\circ}$ to $\mathrm{I}_{200^{\circ}}$ have been plotted and the resulting curves are shown in Plate II. Curve $\left(A^{\prime}\right)$ represents Berthelot's values; curve $\left(B^{\prime}\right)$, those computed by the present writer; curve $\left(C^{\prime}\right)$, Callendar's values. 'The agreement of the three curves is eminently satisfactory, and their similarity indicates that 
TABLE XIII.

Constant-Volume Corrections for Nitrogen, $\mathrm{p}_{\mathrm{o}}=1000 \mathrm{~mm}$.

\begin{tabular}{|c|c|c|c|}
\hline I & 2 & 3 & 4 \\
\hline$t^{\circ} \mathrm{C}$ & Callendar, II & D. Berthelot & E. B. \\
\hline-100 & +0.080 & +0.125 & $(-0.059)$ \\
\hline+50 & -0.0059 & -0.0086 & -0.0014 \\
\hline 200 & +0.035 & +0.046 & +0.024 \\
\hline 400 & $\ldots$ & +0.194 & +0.139 \\
\hline 450 & +0.189 & 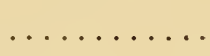 & \\
\hline 600 & & $\ldots \ldots \ldots$ & +0.305 \\
\hline 800 & ........ & +0.56 & +0.514 \\
\hline 1000 & +0.646 & +0.77 & +0.734 \\
\hline 1200 & & & +0.961 \\
\hline
\end{tabular}

a considerable extrapolation by the same methods would be possible before the divergence became of any moment. When we consider that the experimental uncertainty of the melting point of gold at approximately $1065^{\circ}$ is generally estimated to be about $2^{\circ}$, we see that the differences between the corrections as computed by the three methods are altogether insignificant.

As in the case of the constant-pressure corrections, it seems probable that we may regard the thermodynamic corrections of the centigrade, constant-volume, nitrogen scale as known with a precision far exceeding the precision as yet attainable in gas thermometry at these high temperatures.

\section{CORRECTIONS OF THE CONSTANT-VOLUME HYDROGEN THERMOMETER.}

The application to hydrogen of the method just used for nitrogen can not give satisfactory rest1ts because, as has been shown in §I 3 , the constant-pressure corrections for hydrogen appear very uncertain. We shall merely give the differences between the constant-volume and constant-pressure scales as computed by the methods of $\S$ I 6 and $\S$ I 7 .

Chappuis found from his experiments on hydrogen

$$
\underset{23835-07-9}{\left[\frac{\partial}{\partial p}(p v)\right]_{0}}=+0.000762 ;\left[\frac{\partial}{\partial p}(p v)\right]_{100}=+0.000797
$$


If we set $\theta_{0}=273.06$, the equation for the compressibility of hydrogen at low pressures, as derived from the equation of Clausius. becomes

$$
K_{t}=\frac{273.06}{273.06+t}\left[0.0008363-\frac{5.5410}{(273.06+t)^{2}}\right]
$$

When these coefficients, namely,

$$
\begin{aligned}
& \frac{a}{R}=5.5410 \\
& b=0.0008_{3} 63
\end{aligned}
$$

are used as before to compute the value of the critical pressure from the equation of Clausius, on the assumption that the critical temperature is $\theta_{c}=32^{\circ}$, we have

$$
p_{c} \text { (atmospheres) } \begin{array}{ccc}
\text { Calculated } & \text { Observed } \\
\text { I } 3.2 & \text { I } 3-\text { I } 9.4
\end{array}
$$

Chappuis' experiments therefore indicate the lower value as the nnore probable, assuming the validity of the equation of Clausius.

\begin{tabular}{|c|c|c|c|c|}
\hline I & 2 & 3 & 4 & 5 \\
\hline$t^{\circ} \mathrm{C}$ & D. Berthelot & Callendar, I & Callendar, II & E. B. \\
\hline-240 & +1.188 & $\ldots \ldots \ldots$ & $\ldots \ldots \ldots$ & +1.176 \\
\hline-200 & +0.190 & +0.636 & +0.237 & +0.200 \\
\hline-150 & $\ldots \ldots$ & +0.158 & +0.072 & +0.063 \\
\hline-100 & +0.013 & +0.046 & +0.024 & +0.014 \\
\hline-50 & $\ldots \ldots \ldots \ldots$ & +0.013 & +0.006 & $\ldots \ldots$ \\
\hline+50 & -0.0006 & -0.0022 & -0.0012 & -0.000 \\
\hline+200 & +0.002 & +0.009 & +0.007 & +0.003 \\
\hline+300 & $\ldots \ldots$ & +0.022 & +0.016 & +0.008 \\
\hline+400 & +0.009 & & $\ldots \ldots \ldots \ldots$ & +0.011 \\
\hline+450 & & +0.042 & +0.032 & \\
\hline
\end{tabular}

\section{TABLE XIV.}

Difference between Constant-Volume and Constant-Pressure Scales for Hydrogen, $t_{v}-t_{p}, \pi=p_{o}=1000 \mathrm{~mm}$. 
By means of equations (66) and (77), values of $t_{v}-t_{p}$, have been computed for several temperatures. The results are shown in Table XIV. Column I gives the temperature; column 2, the values of $t_{v}-t_{p}$ according to Berthelot; column 3 , the values according to Callendar's first method of computing; column 4 , the values according to his second method; column 5 , the values computed by the writer.

While the discrepancies between columns 2, 4, and 5 are large, in per cent, the absolute values are so small as to indicate that great experimental precision would be needed to detect any difference of run between the two scales except at the lowest temperatures. The fairly close agreement between Berthelot's values and those in column 5 is a necessary consequence of the approximate identity of the two equations of state upon which the computations were based.

\section{CONCLUSION AND SUMMARY.}

The time seems to be approaching when, in thermometry of precision, a mere reference of temperatures to "the gas scale" will be insufficient. The "normal scale" serves very well for low and moderate temperatures, but since it can not in practice be used at all for high temperatures we must soon, to secure uniformity of definition, adopt a different standard, and Lord Kelvin's scale is obviously the one indicated. This is especially true, because it is in fact already the only one in practical use at very high temperatures. For the radiation formulas which serve as a basis for all measurements of such temperatures are derived, in so far as they have any theoretical foundation, from the second law of thermodynamics, and the "temperature" which occurs in the equations used is therefore, by definition, the absolute thermodynamic temperature.

In the foregoing pages the attempt has been made, first, to set forth the relations of the actual gas scales to the thermodynamic scale in such simple form as to be readily available to anyone interested in the subject; second, by new computations based on the best available data, and by comparison with the results obtained by others, to show what our actual knowledge of the relation of existing gas scales to the thermodynamic scale is; and third, to point out, by the way, the particulars in which our experimental knowledge is most 
deficient and the directions in which new experiments are most needed.

The contents of the paper may be summarized as follows:

(I) After a short statement of the principles of gas thermometry, with definitions of the scales used and of Lord Kelvin's thermodynamic scale $(\S \mathrm{I})$, the theory of the constant-volume and constantpressure thermometers, as based on the Joule-Thomson effect, has been developed in the nsual manner, emplasis being laid on the different nature of the experimental data most suitable for connecting the two scales with the thermodynamic scale $(\S \S 2-4)$.

(2) The best available experimental data on the specific volumes, specific heats, and coefficients of expansion have been quoted $(\$ 5$ ).

(3) The numerical values of the Joule-Thomson effect have been examined ( $\$ 6)$, and it has been shown that by the use of the law of corresponding states all the values may be coordinated and represented by a single empirical equation, from which, by an inverse process, values may be obtained which are more probably exact than any that can be deduced from the observations on each gas considered separately $(\S 7)$. This empirical equation has been compared with the empirical equation of Rose-Innes, and with the equation of D. Berthelot, also obtained by the aid of the law of corresponding states $(\S 8)$.

(4) The empirical equation for $\mu$, as well as several simpler approximations, have been utilized in integrating the equations of the constant-pressure thermometer ( $(9)$. The thermodynamic temperature of the melting point of ice has been computed in several ways, and the resulting values compared with one another and with the values obtained by an altogether independent method, by D. Berthelot (§ IO).

(5) Valnes have been computed for the thermodynamic corrections of the constant-pressure hydrogen and nitrogen thermometers between the ice and steam points, and compared with the values computed by Rose-Innes, Callendar, and D. Berthelot ( $\S$ I I).

(6) Corrections for the same scales have been computed for larger ranges of temperature and compared with the values obtained by Callendar and D. Berthelot. The lack of concordance in the case of hydrogen indicates the need of further experiments on this gas. With nitrogen the agreement of the results of the different methods 
of computation is satisfactory ( $\left.\S \mathrm{I}_{2}, \mathrm{I} 3\right)$. The corrections have been tested by means of the determinations of the boiling point of sulphur by Callendar and Griffitlıs, and by Cliappuis and Harker, with satisfactory results. The centigrade thermodynamic temperature of this point appears to be close to $444^{\circ} \cdot 9$ ( I 4 ).

(7) The relation of the constant-pressure and constant-volnume scales has been discussed ( $\left.\S I_{5}, I 6\right)$. The differences of the two scales have been computed for nitrogen, and hence the thermodynanic corrections of the constant-volume nitrogen scale deduced from those of the constant-pressure scale already found. Comparison with the results of Callendar and D. Berthelot shows a very close agreement $(\S \mathrm{I} 7)$. Similar computations and comparisons in the case of hydrogen show merely that the thermodynamic corrections of the constant-volume hydrogen thermometer are in most, if not all, cases negligible, the corrections, while uncertain, being small ( $\S$ I 8 ).

Washington, February 4, 1907. 